\title{
Cortical Areas Involved in OKN and VOR in Cats: Cortical Lesions
}

\author{
Ronald J. Tusa, ${ }^{1}$ Joseph L. Demer ${ }_{i}^{2}$ and Susan J: Herdman ${ }^{3}$ \\ 'Department of Ncurology, Johns Hopkins Hospital, Baltimore, Maryland 21205, 2Julcs Stein Eye Institute and Department \\ of Neurology, UCLA, Los Angeles, California 90024, and 'Department of Otolaryngology-Head and Neck Surgery, Johns \\ Hopkins Hospital, Baltimore, Maryland 21205
}

Eye movements evoked by optokinetic and vestibular stimulation were measured by scleral search coil before and up to 8 weeks after unilateral cortical lesions in 11 cats. During both monocular and binocular viewing conditions, several deficits in the velocity-storage component of optokinetic nystagmus (OKN) were found. At low target velocities, the final steady-state slow-phase eye velocity and the peak value of optokinetic afternystagmus (OKAN) were reduced for slow phases towards the side of the lesion. At high target velocities OKN and OKAN were no longer elicited. The time constant of OKAN was also reduced for slow phases towards the side of the lesion. These deficits in OKN and OKAN were quantitatively similar in cats with large cortical (LC) lesions involving all known visual areas and in cats with suprasylvian (SS) lesions involving areas 21a, 21b, PMLS, and VLS. Ablation of areas 17 and 18 alone had no effect, but when combined with section of the corpus callosum $(17 / 18+\mathrm{CC})$ resulted in a qualitatively similar but less severe deficit as the LC and SS lesions. By 3 weeks postoperatively, OKN recovered to near preoperative values in cats with SS lesions.

Vestibular adaptive capabilities were impaired during the duration of the study in cats with LC, SS, and 17/18+CC lesions. Cats with these lesions could not normally increase VOR gain for slow phases directed ipsilateral to the lesion, and following vestibular adaptation most of these cats developed persistent asymmetries in VOR gain and VOR time constants.

These results can be better conceptualized using a mathematical model of the vestibulo-ocular and optokinetic system adapted from Robinson (1977). This model contains a single positive-feedback velocity storage loop common to the VOR and OKN systems and a retinal-slip velocity nonlinearity. Our results suggest that SS cortex improves the retinal-slip nonlinearity feeding into the velocity-storage system by extending its range and increasing its gain. The SS cortex depends in part upon areas 17 and 18 either directly,

\footnotetext{
Received Feb. 10, 1988; revised Sept. 7, 1988; accepted Sept. 8, 1988.

This work was supported by the Sloan Foundation, NIH Grants NS00804 and EY06394, and the Wilmer Core Grant EY-01765. We thank Drs. David Zee and Tim Hain for their comments on the manuscript and Dr. James Sprague for doing the area $17 / 18$ surgical ablations and for giving us advice on testing visual field and placing responses. We also thank Corena Bridges for art work and Cathy Bonolis for manuscript preparation. We also appreciate the technical help from the Wilmer Biostatistical Center supported by NIH Grant EY01765.

Correspondence should be addressed to Ronald J. Tusa, M.D., Ph.D., Department of Neurology, Meyer 2-147, Johns Hopkins Hospital, 600 N. Wolfe Street, Baltimore, MD 21205.
}

Copyright (C) 1989 Society for Neuroscience 0270-6474/89/041 163-16\$02.00/0 or indirectly via the corpus callosum, for processing of high retinal-slip velocities. Cerebral cortex is also involved in increasing the gain of the velocity-storage loop during vestibular adaptation for ipsilaterally directed slow phases.

In the previous study (Herdman et al., 1989), we demonstrated that only areas 17 and 18 , and four suprasylvian areas (PMLS, VLS, $2 \mathrm{la}$ and $2 \mathrm{lb}$ ) had an increase in metabolic activity in cats gencrating optokinctic nystagmus (OKN) compared with cats viewing a stationary $\mathrm{OKN}$ drum. In this study we examined vestibular and optokinetic eye movements in cats with unilateral lesions restricted to these cortical areas compared to cats with unilateral lesions involving all known visually responsive cortex. We examined the effects of these lesions on the range of retinal-slip velocities (OKN drum velocity minus slow-phase eye velocity) that can drive OKN, the gain and the lime constants of the optokinetic and vestibular response, and VOR gain adaptation.

We postulate that the suprasylvian (SS) areas (PMLS, VLS, $21 \mathrm{a}$, and $21 \mathrm{~b}$ ) are the cortical areas of a corticosubcortical pathway involved in generating ipsilateral optokinetic slow phases and that areas 17 and 18 comprise the major source of visual input to these SS areas for OKN. Areas PMLS, 2la, and $21 \mathrm{~b}$ project strongly to the dorsal terminal nucleus, a structure in the midbrain that is involved in horizontal OKN (Berson and Graybiel, 1980; Marcotte and Updyke, 1982). Areas PMLS and $21 \mathrm{a}$, in turn, receive a bilateral projection from areas 17 or 18 (Segraves and Rosenquist, 1982). The findings from previous behavioral studies in cats following unilateral and bilateral lesions of areas 17-19, and unilateral lesions of cortex in the SS gyrus and sulcus are consistent with this concept (Montarolo et al., 1981; Hoflmann, 1982; Strong et al., 1984; Ventre, 1985), but no study has examined the effects of selective ablation of area PMLS, VLS, 21a, and 21b compared with large cortical lesions involving all known visual areas to determine whether these are the critical areas involved in OKN. In addition, no study has examined the effects of visual deafferentation of these SS areas from unilateral 17 and 18 lesions paired with section of the corpus callosum.

Finally, the contribution of areas 17, 18, PMLS, VLS, 21a, and $21 \mathrm{~b}$ on VOR gain adaptation is unknown. Although visual deprivation studies suggest that VOR gain adaptation depends upon cerebral cortex (Harris and Cynader, 1981), VOR gain adaptation has not been specifically examined in cats following cortical lesions.

Preliminary reports of these experiments have been presented elsewhere (Demer et al., 1983; Tusa et al., 1983). 

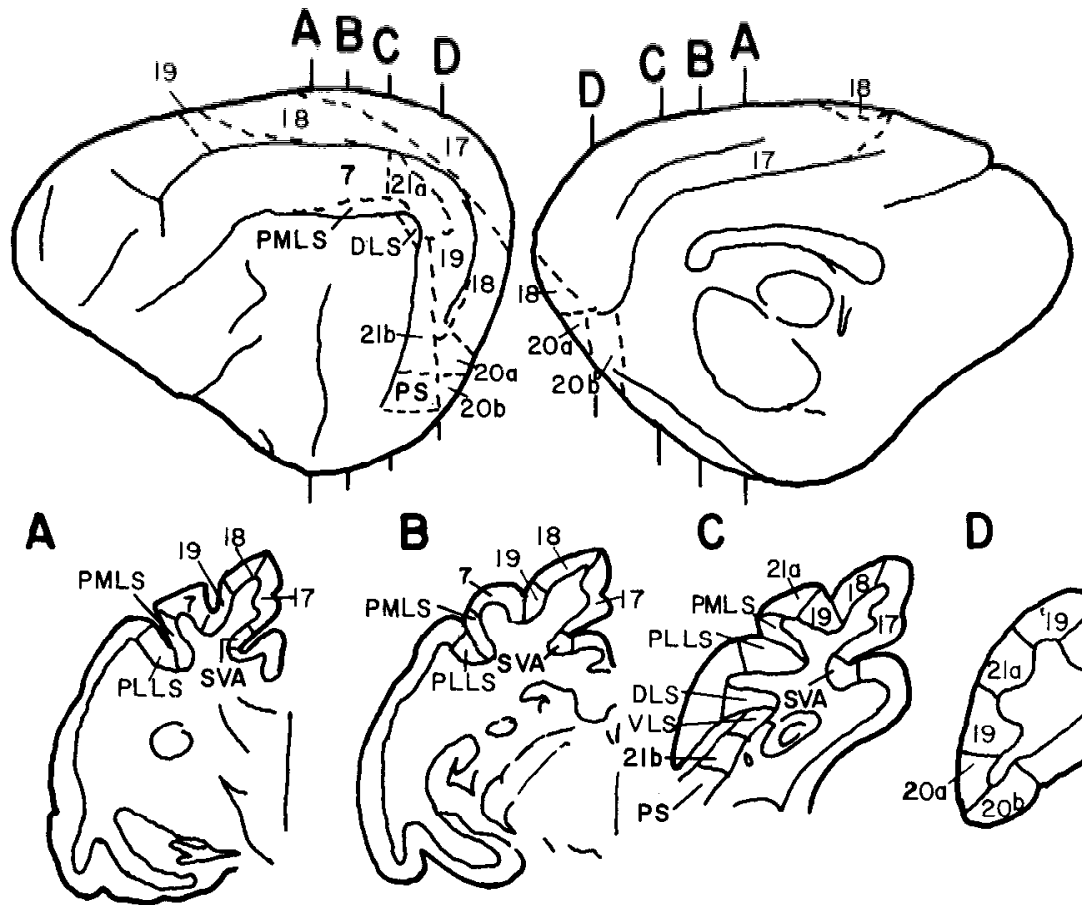

D

Figure 1. Dorsolateral and medial surface views of the cat brain and selected coronal sections showing the location of the cortical visual areas involved in this study. This figure is based on the maps published by Rosenquist (1985).

\section{Materials and Methods}

\section{Behavioral testing}

Eleven adult male cats were used in this study. Eye movements were measured using the magnetic-field search coil technique in head-stabilized cats. The details of calibrating and recording eye movements using this technique were described in the previous paper (Herdman et al., 1989). The cat's visual field, visual placing, and tactile placing responses were tested pre- and postoperatively (see Sprague and Meikle, 1965, for details). The mean spontaneous eye drift velocity was determined in the dark and also in the light while the animals viewed a stationary optokinetic drum.

Optokinetic responses. Optokinetic responses were elicited inside a rotating full-field drum containing a random pattern of black circles each subtending a visual angle of $8^{\circ}-16^{\circ}$ on a white background. The drum was rotated at a constant velocity for $60 \mathrm{sec}$. Both the initial rise in slow-phase eye velocity during the first second (mediated by a quick-responding or pursuit component of $\mathrm{OKN}$ ) and the slow rise in slow-phase eye velocity to a steady-state value (mediated by the velocity-storage component of $\mathrm{OKN}$ ) were examined. To determine mean steady-state eye velocity, the average value of each of the last 5 consecutive slow phases was measured, and these values, in turn, were averaged. At the end of $60 \mathrm{sec}$, the light was turned off to record optokinetic afternystagmus (OKAN). Maximum OKAN and the OKAN time constants, the time for slow-phase eye velocity to decay to $37 \%$ of its maximum value, were determined. Since there is no immediate decrease in slow-phase eye velocity when the light is turned off in cats, the first slow-phase eye velocity generated in the dark was used as the maximum OKAN. OKN slow-phases were measured both during binocular and during monocular viewing at drum speeds ranging from $5^{\circ}$ to $120 \% \mathrm{sec}$. After OKN was recorded at each drum speed, the entire slow-phase eye velocity response in each cat was described by 3 measurements: (1) the peak or maximum steady-state eye velocity achieved; (2) retinal-slip velocity associated with peak steady-state eye velocity; and (3) maximum gain. Gain (steady-state eye velocity/OKN drum velocity) was measured at each drum speed, and the mean value of the top 3 values was listed as the maximum gain.

Vestibular responses. The cat chair and the magnetic field coils were rotated en bloc in the dark either sinusoidally $(30 \% \mathrm{sec}$ peak velocity at 0.05 and $0.25 \mathrm{~Hz})$ or by constant-velocity steps $\left(30^{\circ}\right.$ and $\left.60^{\circ} / \mathrm{sec}\right)$ to elicit vestibular nystagmus. Velocity of the chair was measured by a tachometer. Gain (peak slow-phase eye velocity/peak chair velocity) of the vestibular response both for steps and for sinusoids of chair rotation in the dark was determined. The mean response of 6 trials in each direction was then calculated. Symmetry of responses was quantitated using an index $(I)$,

$$
I=|| \frac{G L-G R}{G L+G R}||
$$

where $G L$ and $G R$ are the mean gain to the left and right, respectively, for similar stimuli. The VOR time constant, the time for eye velocity to decay to $37 \%$ of its maximum value, was also determined for the VOR step responses.

Visual-vestibular interactions and VOR gain adaptation. Visual-vestibular interactions were measured by sinusoidally rotating the VOR chair and $\mathrm{OKN}$ drum at $0.05 \mathrm{~Hz}, 30 \% \mathrm{sec}$ peak velocity in the light, with the chair and drum either in phase (X0 viewing) or $180^{\circ}$ out of phase (X2 viewing). VOR gain adaptation, the degree to which VOR gain in the dark was changed after prolonged $\mathrm{X} 2$ or $\mathrm{X} 0$ viewing, was also tested. During these adaptation testing sessions, an attempt was made to increase the VOR gain by exposing the cats to X2 viewing for $120 \mathrm{~min}$, and then an attempt was made to reduce the VOR gain by exposing the cats to $\mathrm{X} 0$ viewing for $120 \mathrm{~min}$. To determine the change in VOR gain, VOR gains were measured in the dark before and after each prolonged $\mathrm{X} 2$ or $\mathrm{X} 0$ viewing session.

\section{Surgery}

After behavioral testing was completed, the cats were anesthetized with intravenous sodium thiopental and a craniotomy was performed over one hemisphere. Using subpial aspiration techniques 1 of 4 different lesions was performed on each cat. In 4 cats, all known visually responsive cortical areas in the occipital, temporal, and parietal lobes were removed (Tusa et al., 1981; Rosenquist, 1985; Fig. 1). In 3 cats, areas PMLS, VLS, $21 \mathrm{a}$, and $21 \mathrm{~b}$ were removed. In 4 cats, areas 17 and 18 were removed, either in isolation or in combination with section of the corpus callosum. After surgery, the craniotomy was closed with a dental cement cap, muscle, and skin. Corticosteroids and antibiotics were given for 1 week. Postoperatively, the cats were tested behaviorally within $3 \mathrm{~d}$ of the surgery, and then weekly for 6 weeks.

After postoperative testing, the animals were given a lethal injection of intravenous phenobarbital and perfused with saline followed by buffered $10 \%$ formalin. The brains were removed, photographed, and serially sectioned in coronal $40 \mu \mathrm{m}$ sections. Every 10 th section was stained 


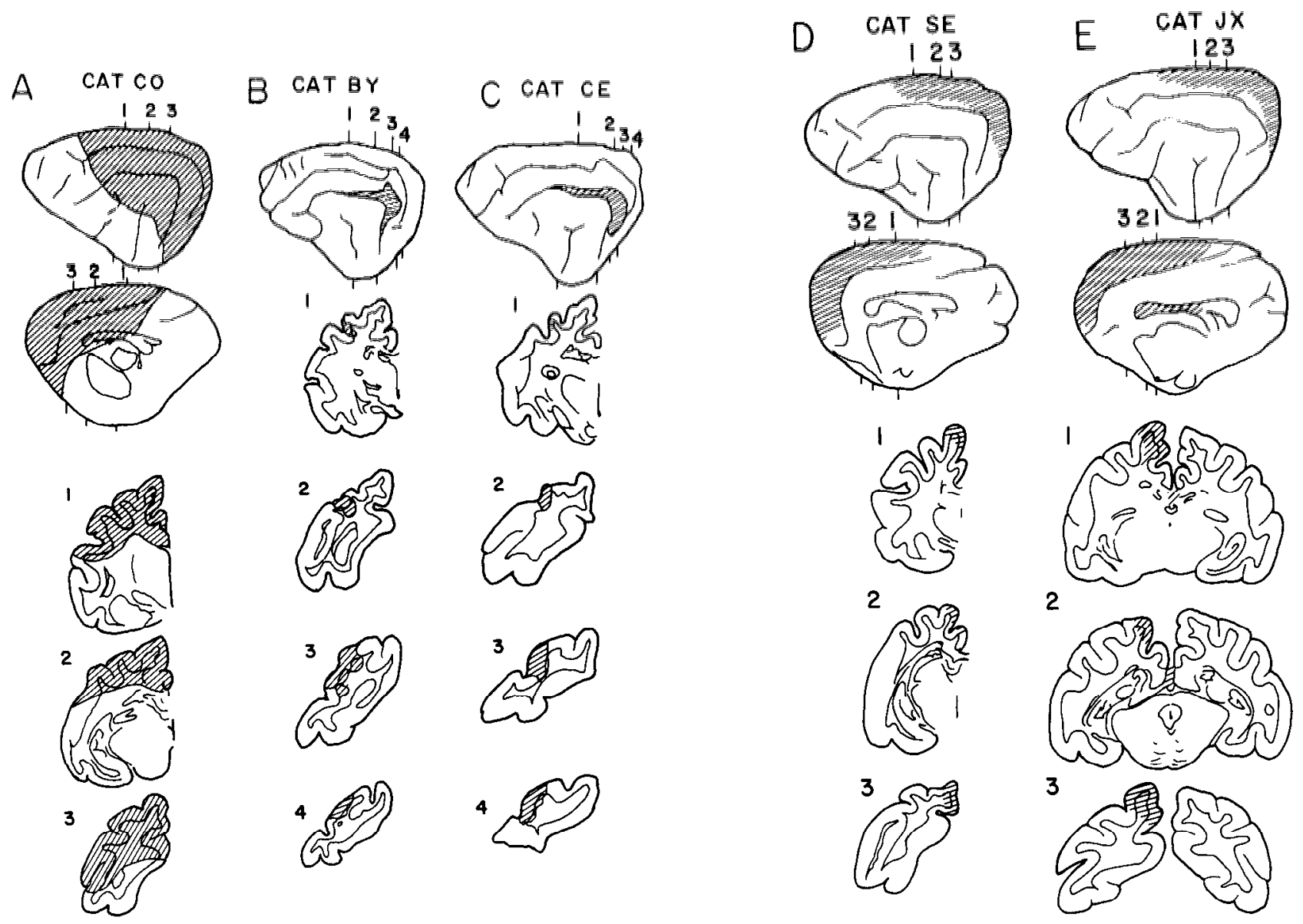

Figure 2. Histological reconstruction of cortical lesions in one or more representative cats from each lesion group; large cortical lesion ( $A$ ), SS lesion $(B$ and $C$ ), 17 and 18 lesion $(D)$, and 17 and 18 plus corpus callosum lesion $(E)$.

with a cresyl violet cell stain and every 20 th section stained with a myelin stain.

\section{Data analysis}

Data were recorded on a chart recorder and stored on FM tape for subsequent analysis (overall bandwidth, $1 \mathrm{kHz}$ ). Slow-phase eye velocity was determined from analog differentiation of eye position (bandwith cutoff, $5.0 \mathrm{~Hz}$ ).

Eye movement traces were monitored on-line to detect signs of decreased alertness, manifested by distortion of the saccadic or quickphase waveform. Recordings were stopped if the animal showed signs of fatigue. Cat alertness during the prolonged $\mathrm{X} 2$ or $\mathrm{X} 0$ viewing session was maintained by intramuscular dextroamphetamine sulfate $(0.5 \mathrm{mg} /$ $\mathrm{kg}$ ). During the other testing sessions, cat alertness was maintained using novel noise and tactile stimulation.

Based on 3 separate preoperative measurements, the $99 \%$ confidence intervals of normal variation in the cat's OKN and VOR responses were determined. All postoperative values that fell outside of the $99 \%$ confidence intervals were labeled as statistically significant.

\section{Results}

\section{Extent of lesions}

Reconstructions of the lesion for representative animals are shown in Figure 2. In cats with the large cortical (LC) lesions, all known visually responsive areas, except for the frontal eye fields, were either ablated or damaged (Fig. $2 A$ ). The visually responsive areas involved in this lesion included areas 7 and 17-21, areas within the SS sulcus (AMLS, ALLS, PMLS, PLLS, VLS, DLS), splenial visual area (SVA), and the posterior su- prasylvian area (PS). The lesion spared the basal ganglia and thalamus.

Reconstructions of a lesion involving SS cortex in 2 cats are shown in Figure 2, $B, C$. This lesion primarily involved areas PMLS, $21 \mathrm{a}$, and $21 \mathrm{~b}$ and minimally involved areas VLS and AMLS. In cat BY the lesion extended into the underlying white matter, whereas in cat CE it was primarily confined to the cortex. Although not shown, cells in laminae A and Al of the dorsal lateral geniculate nucleus (dLGN) were spared, but there was cell loss in laminae $\mathrm{C}$ consistent with the projection of this part of dLGN to the lesioned cortex.

Reconstructions of a lesion involving areas 17 plus 18 , and a lesion involving areas 17 and 18 plus section of the corpus callosum are shown in Figure 2, $D, E$, respectively. Areas 17 and 18 were completely removed rostrally, and only the most ventral aspect of area 17 on the tentorial surface was spared. This portion of cortex contains the most peripheral parts of the visual field $\left(>60^{\circ}\right)$. Cells in the most caudal parts of laminae $A$ and A1 of the dLGN were also intact in these animals, reflecting the sparing of this portion of area 17.

\section{General observations}

Following the LC lesion there was a persistent visual field deficit contralateral to the lesion during perimetry testing. There was no visual placing contralateral to the lesion, but tactile placing was present. Cats with this lesion had a spontaneous nystagmus 


\section{A OKN Left}
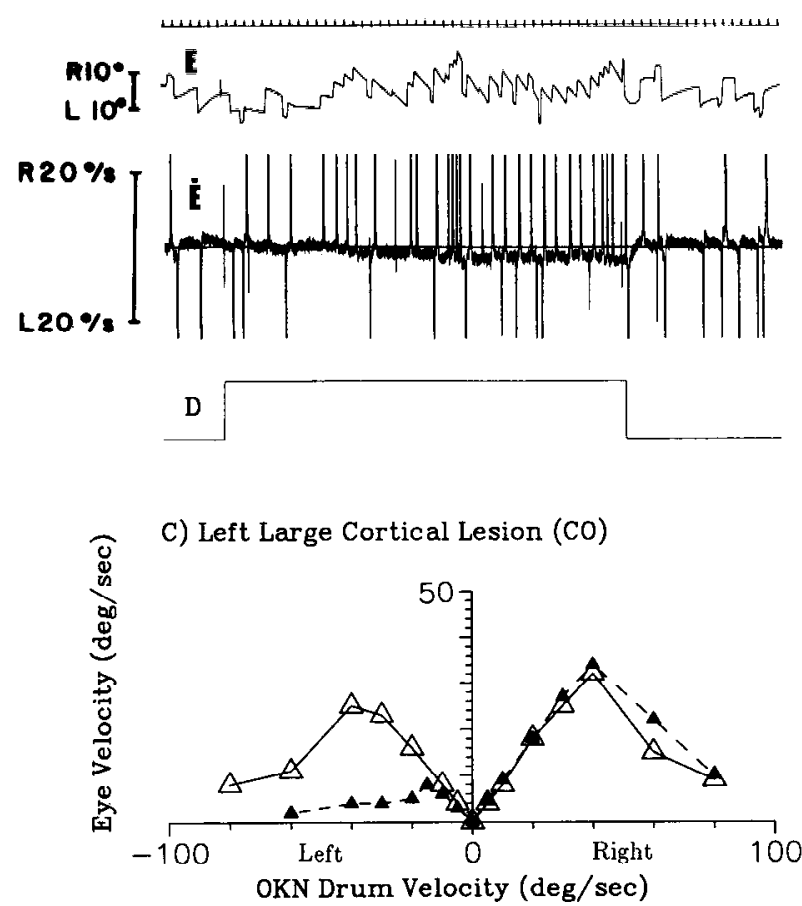
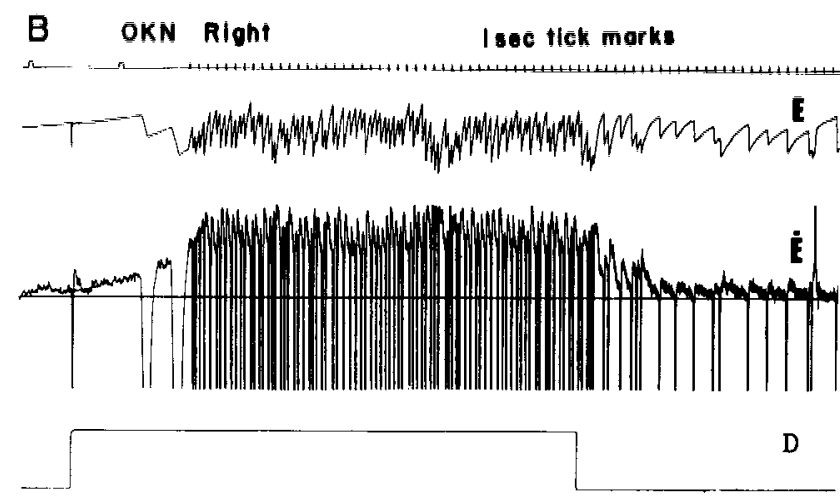

D)

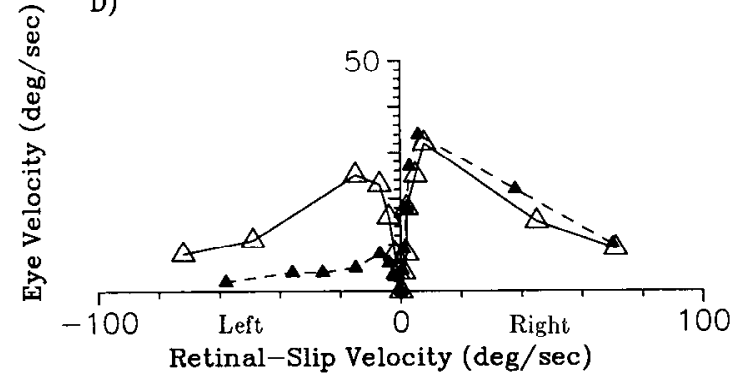

Figure 3. OKN 3 weeks following a left-sided large cortical lesion in cat CO. Responses for a $20 \%$ sec drum rotation towards $(A)$ and away from $(B)$ the side of the lesion are shown. Eye position $(E)$, eye velocity $(E)$, and $\mathrm{OKN}$ drum light $(D)$ are illustrated. OKN responses measured at several different drum velocities are plotted as steady-state eye velocity against OKN drum velocity $(C)$ and steady-state eye velocity against retinal-slip velocity $(D)$. Open triangles represent preoperative values, and closed triangles represent postoperative values. The response was decreased towards the lesioned side, especially at high retinal-slip velocities.

with a $1^{\circ}-2 \%$ sec slow-phase eye velocity drift away from side of the lesion that was present both in the light and dark. This nystagmus persisted for 3-7 d. When aroused, these cats had head turning toward the lesioned side, which persisted for several weeks.

Cats with SS cortical lesions also had a spontaneous nystagmus similar to cats with the LC lesions. These cats had normal visual fields during perimetry testing.

Cats with the 17/18 plus corpus callosum lesions showed an initial visual field deficit during perimetry testing during the first postoperative month. They turned their heads towards the side of the lesion, although only during the first postoperative week. These cats also had spontaneous nystagmus similar to cats with the LC lesions. The contribution of spontaneous nystagmus to $\mathrm{OKN}$ and VOR gain measurements in these cats, and all other cats with cortical lesions, was eliminated. This was done by subtracting the spontaneous slow-phase velocity for VOR and OKN slow phases directed away from the lesion and adding the spontaneous velocity for VOR and OKN slow phases directed towards the lesion.

Cats with the 17/18 lesions also had a visual field defect during the first postopcrative month. Thesc cats had no spontaneous nystagmus or head turning.

\section{Optokinetic responses}

The quick-responding ("pursuit") component of OKN could not be reliably assessed. This was because this component was inconsistent and usually $<5 \% \mathrm{sec}$. Also, there was no sudden drop in eye velocity when the lights were turned off, which would reflect the termination of the quick-responding component.
Following ablation of cortex, there were 3 deficits in the velocity-storage component of OKN. First, the gain (eye velocity/ drum velocity) of steady-state OKN and the peak OKAN value after the lights were turned off were decreased at all drum velocities. Second, there was a reduction in the upper range of retinal-slip velocitics that could maximally drive the velocity storage component of OKN. Third, the time constant of OKAN was decreased, especially for slow phases directed towards the side of the lesion. These deficits in steady-state eye velocity and the time constant of the OKAN will be further described.

\section{Steady-state eye velocity}

Steady-slate eye velocity of optokinetic slow phases generated toward the side of the lesion was reduced during both binocular and monocular viewing in cats with LC, SS, and the $17 / 18$ plus corpus callosum lesions. Optokinetic slow phases were not affected in cats with the 17/18 lesion in either binocular or monocular viewing conditions. Figure 3 illustrates the $\mathrm{OKN}$ response 3 weeks after a left-sided LC lesion. For a $20 \% \mathrm{sec}$ optokinetic drum rotation towards the side of the lesion, steady-state eye velocity was $4 \% \mathrm{sec}$ (Fig. $3 A$ ); drum rotation away from the side of the lesion elicited an eye velocity of $18 \% \mathrm{sec}$ (Fig. $3 B$ ). When steady-state eye velocity was measured at several optokinetic drum speeds, the decrease in slow-phase velocity was found at all drum speeds (Fig. $3 C$ ). The same data plotted against retinalslip velocity shows that maximal $\mathrm{OKN}$ responses occurred at lower retinal-slip velocities after the lesion (Fig. 3D).

Steady-state eye velocity plotted against retinal-slip velocity in one representative cat from each of the 4 lesion groups is shown in Figure 4. Both binocular and monocular viewing con- 
A) Left Large Cortical Lesion (GY)

Binocular Viewing

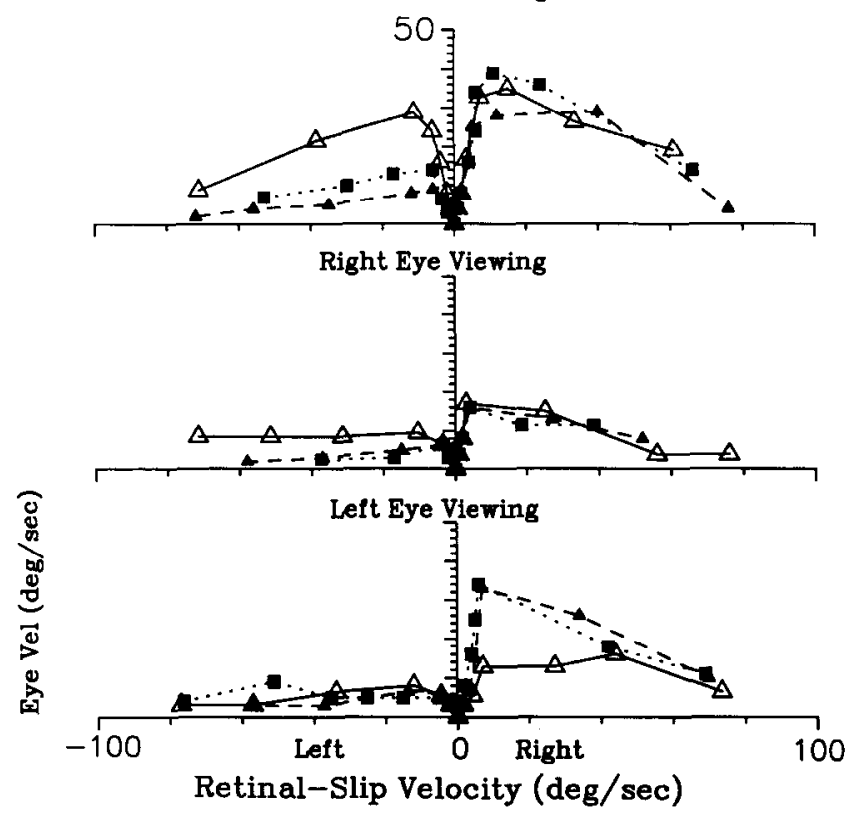

C) Left 17/18 + Corpus Callosum Lesion (BE) Binocular Viewing

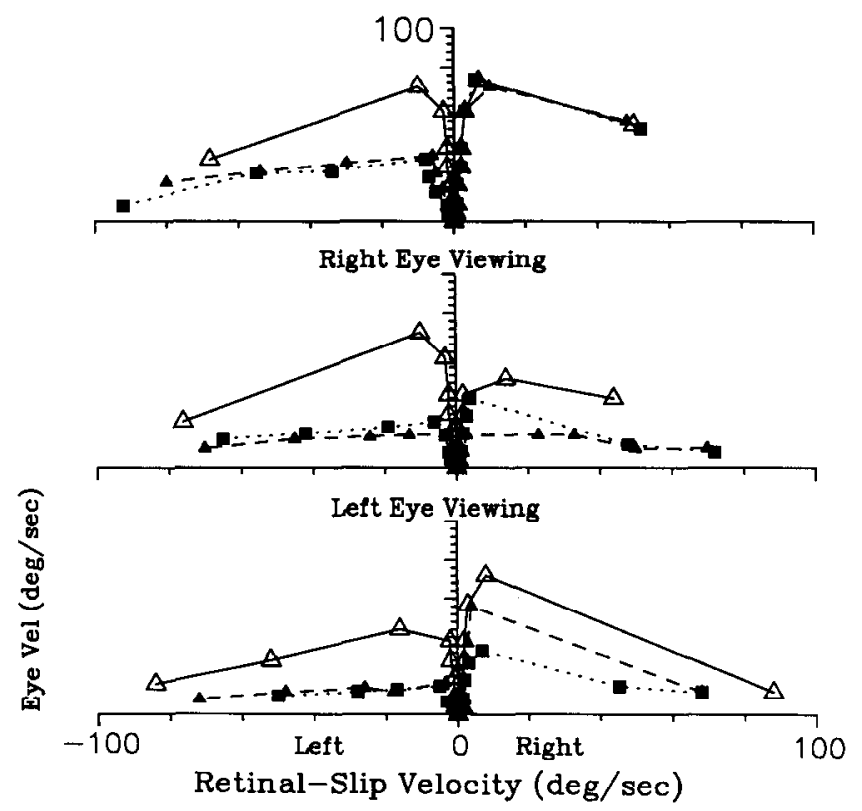

B) Left Suprasylvian Lesion (BY) Binocular Viewing
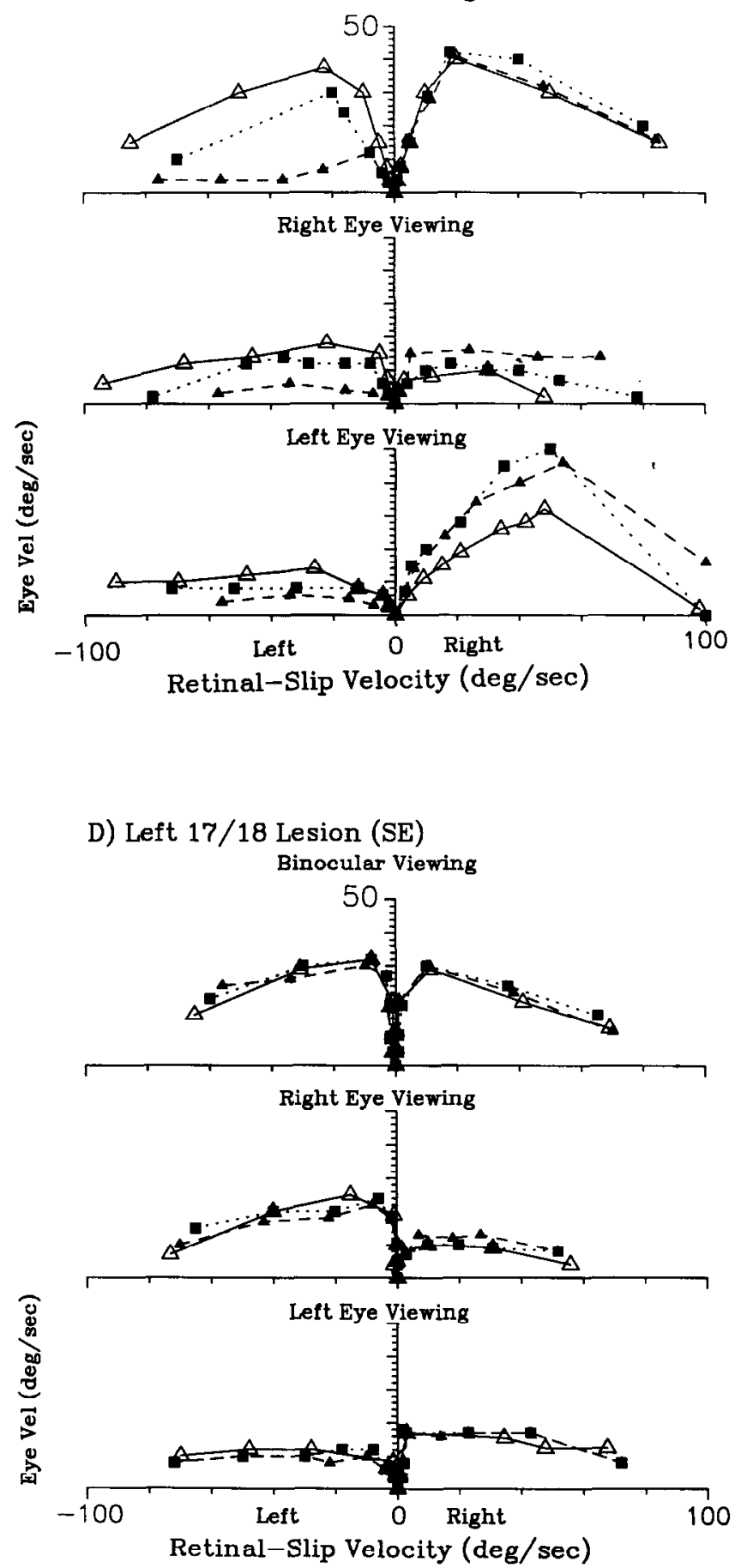

Figure 4. OKN responses in one cat from each group: $A$, Left-sided LC lesion; $B$, left-sided SS lesion; $C$, left-sided $17 / 18$ plus corpus callosum lesion; $D$, left-sided $17 / 18$ lesion. Steady-statc cyc vclocity is plottcd on the ordinatc and retinal-slip velocity on the abscissa. Measurements were made preoperatively (open triangles), $3 \mathrm{~d}$ postoperatively (closed triangles), and 3 weeks postoperatively (closed squares). OKN during both binocular and monocular viewing conditions is shown. Postoperatively, slow-phase eye velocities to the left (towards lesion) were initially reduced in all groups except the $17 / 18$ lesion group. This was true for both binocular and monocular viewing. Responses recovered in cats with the SS lesion. Postoperatively, slow-phase eye velocity to the right (away from the lesion) was greater than preoperatively in some cats. This was most marked during left eye viewing in cats with LC and small SS lesions.

ditions are shown preoperatively, as well as $3 \mathrm{~d}$ and 3 weeks postoperatively. Three days postoperatively, cats with LC, SS, and $17 / 18$ plus corpus callosum lesions had reductions of peak eye velocities towards the side of the lesion, measured during both binocular and monocular viewing conditions. By 3 weeks postoperatively, slow-phase eye velocities of cats with the SS lesion recovered to near preoperative values. In contrast, slowphase eye velocities showed no to little recovery in cats with LC or 17/18 plus corpus callosum lesions. Although not illustrated, peak slow-phase eye velocity during OKAN was similarly 
Table 1. Summary of OKN responses for slow phases directed towards the side of the lesion measured preoperatively and $3 \mathrm{~d}$ and 3 weeks postoperatively

\begin{tabular}{|c|c|c|c|c|c|c|c|c|c|}
\hline \multirow[b]{2}{*}{ Cat } & \multicolumn{3}{|c|}{ Pre-op } & \multicolumn{3}{|c|}{3 days post-op } & \multicolumn{3}{|c|}{3 weeks post-op } \\
\hline & $\begin{array}{l}\text { Peak } \\
\text { Evel } \\
\% \text { s } \\
\end{array}$ & $\begin{array}{l}\text { Ret- } \\
\text { slip } \\
\% \text { s }\end{array}$ & $\begin{array}{l}\text { Max } \\
\text { gain }\end{array}$ & $\begin{array}{l}\text { Peak } \\
\text { Evel } \\
\% \text { s } \\
\end{array}$ & $\begin{array}{l}\text { Ret- } \\
\text { slip } \\
\% / \mathrm{s}\end{array}$ & $\begin{array}{l}\text { Max } \\
\text { gain }\end{array}$ & $\begin{array}{l}\text { Peak } \\
\text { Evel } \\
\% \text { s }\end{array}$ & $\begin{array}{l}\text { Ret- } \\
\text { slip } \\
\% / \mathrm{s}\end{array}$ & $\begin{array}{l}\text { Max } \\
\text { gain }\end{array}$ \\
\hline \multicolumn{10}{|c|}{ Large cortical lesion } \\
\hline GY & 29 & 11 & 0.80 & $9 *$ & 6 & $0.63^{*}$ & $14^{*}$ & 6 & $0.68^{*}$ \\
\hline $\mathrm{CO}$ & 25 & 15 & 0.80 & $8^{*}$ & $7^{*}$ & $0.60^{*}$ & $5^{*}$ & $5^{*}$ & $0.67^{*}$ \\
\hline SR & 54 & 26 & 0.83 & $10^{*}$ & $9 *$ & $0.54 *$ & $18^{*}$ & $12^{*}$ & $0.58^{*}$ \\
\hline SY & 64 & 36 & 0.75 & $10^{*}$ & $7^{*}$ & $0.43^{*}$ & $14^{*}$ & $16^{*}$ & $0.50^{*}$ \\
\hline \multicolumn{10}{|c|}{ Suprasylvian lesion } \\
\hline BY & 38 & 22 & 0.75 & $12^{*}$ & $8^{*}$ & $0.60^{*}$ & 30 & 20 & $0.60^{*}$ \\
\hline $\mathrm{CE}$ & 30 & 15 & 0.84 & $11^{*}$ & $4^{*}$ & 0.78 & 26 & 14 & 0.77 \\
\hline MS & 51 & 19 & 0.85 & $21^{*}$ & $9 *$ & $0.70^{*}$ & 43 & 17 & $0.72 *$ \\
\hline \multicolumn{10}{|c|}{$17 / 18+$ Corpus callosum lesion } \\
\hline $\mathrm{BE}$ & 70 & 10 & 0.95 & $34^{*}$ & $6^{*}$ & $0.81^{*}$ & $32 *$ & 8 & $0.76^{*}$ \\
\hline $\mathrm{JX}$ & 25 & 35 & 0.74 & $13^{*}$ & $17^{*}$ & 0.65 & $12^{*}$ & $18^{*}$ & $0.56^{*}$ \\
\hline TR & - & - & - & - & - & - & $35^{*}$ & $15^{*}$ & $0.68^{*}$ \\
\hline \multicolumn{10}{|c|}{ 17/18 Lesion } \\
\hline TR & 75 & 25 & 0.90 & 69 & 21 & 0.85 & - & - & - \\
\hline SE & 32 & 8 & 0.90 & 30 & 10 & 0.86 & 32 & 8 & 0.85 \\
\hline
\end{tabular}

$99 \%$ Confidence intervals

$$
2.5-10.1 \quad 0.8-7.2 \quad 0.04-0.11
$$

Peak cye velocity (Peak Evel), retinal-slip velocity at peak eye velocity (Ret-slip), and maximum gain (Max gain) for each cat are listed. One cat (TR) received a second-stage corpus callosum section $4 \mathrm{~d}$ after a unilateral $17 / 18$ lesion. Preoperative measurements were repeated several times in order to determine the $99 \%$ confidence intervals of normal variation, which are listed at the bottom of the table. The postoperative measurements that fall outside of these confidence intervals are marked with an asterisk. Three days postoperatively, peak eye velocity, maximum retinal-slip velocity, and maximum gain were significantly reduced in cats with LC, SS, and $17 / 18$ plus corpus callosum lesions. There was no change in cats with the $17 / 18$ lesion. By 3 weeks postoperatively, peak eye velocity and retinal-slip velocity at peak eye velocity recovered in the cats with the SS lesion.

reduced towards the side of the lesion following LC, SS, and $17 / 18$ plus corpus callosum lesions.

There were 2 separate deficits accounting for the reduction in steady-state eye velocity. First, there was a reduction in the range of retinal-slip velocities that could maximally drive OKN, i.e., a reduction in the retinal-slip velocity that elicited the highest steady-state eye velocity before saturation or fall in slow-phase eye velocity occurred. This reduction in range was significant in 3 out of 4 cats with LC lesions, in all cats with SS, and in all cats with $17 / 18$ plus CC lesions (Table 1). Second, there was a reduction in optokinetic gain measured at all OKN drum speeds, which was significant in all cats with LC lesions, in 2 out of 3 cats with SS lesions, and in 2 out of 3 cats with $17 / 18$ plus $\mathrm{CC}$ lesions (Table 1). Table 1 shows peak steady-state eye velocity achieved in response to steps of OKN drum speeds over the range $5^{\circ}-120 \%$ sec; retinal-slip velocity at peak steady-state eye velocity; and maximum gain (steady-state eye velocity/OKN drum velocity). Preoperative measurements were done on 3 separate days in order to determine normal variations about the mean, which are expressed as $99 \%$ confidence intervals at the bottom of the table. These intervals were based on the mean variability in each cat during the 3 preoperative days, which were, in turn, averaged among all 11 cats. For example, in cat GY the peak eye velocity would be expected to range from $18.9^{\circ}$ to $39.1 \% \mathrm{sec} 99 \%$ of the time. The postoperative measurements that fall outside of this confidence interval of normal variability are indicated by an asterisk. Three days postoperatively, OKN responses in cats with $\mathrm{LC}, \mathrm{SS}$, and $17 / 18$ plus $\mathrm{CC}$ fell outside of the $99 \%$ confidence interval of normal variation. Peak steadystate eye velocity decreased to $34 \%$ of preoperative values, peak optokinetic slow-phase eye velocity occurred at retinal-slip velocities that were $42 \%$ of preoperative values. The two cats with a $17 / 18$ lesion had no deficit. By 3 weeks postoperatively, cats with the SS lesion recovered OKN responses to high retinal-slip velocities, but maximum gain was still reduced. Cats with LC and $17 / 18$ plus CC lesions showed little recovery by 3 weeks postoperatively. Of the 3 cats with the $17 / 18$ lesion plus section of the corpus callosum, 2 cats had a single-stage lesion, and 1 cat (TR) had a corpus callosum section $4 \mathrm{~d}$ after a unilateral $17 / 18$ lesion, but all showed a similar deficit. The cats with LC and $17 / 18$ plus CC lesions had still not recovered by 6 weeks postoperatively, which was the last day the cats were examined.

Steady-state eye velocity for optokinetic slow phases generated away from the side of the lesion was increased postoperatively in cats with the $\mathrm{LC}$ and SS lesions (Fig. 4, $A, B$ ). In some cats this occurred during binocular viewing, but it was usually seen only during monocular viewing from the left eye (eye ipsilateral to the lesion). This was primarily due to an increase in maximum gain, which increased by as much as $20 \%$ from preoperative values. In cats with a $17 / 18$ plus corpus callosum lesion, slow-phase eye velocity generated away from the side of the lesion was decreased during monocular viewing.

\section{OKAN time constants}

In Table 2, time constants for slow phases directed towards $(T T)$ and away $(T A)$ from the side of the lesion are shown, along the 


\begin{tabular}{|c|c|c|c|c|c|c|c|c|c|}
\hline \multirow[b]{2}{*}{ Cat } & \multicolumn{3}{|c|}{$\underline{\text { Pre-op }}$} & \multicolumn{3}{|c|}{3 Days post-op } & \multicolumn{3}{|c|}{3 Weeks post-op } \\
\hline & TT & TA & I & TT & TA & I & TT & TA & I \\
\hline \multicolumn{10}{|c|}{ Large cortical lesion } \\
\hline GY & 4.0 & 3.6 & 0.05 & $0.9^{*}$ & 2.1 & $0.40^{*}$ & 3.6 & $5.7^{*}$ & $0.22^{*}$ \\
\hline $\mathrm{CO}$ & 2.5 & 2.8 & 0.05 & $0.8^{*}$ & 1.8 & $0.38^{*}$ & 1.3 & $5.7^{*}$ & $0.63^{*}$ \\
\hline $\mathrm{SR}$ & 4.6 & 5.1 & 0.05 & $1.2^{*}$ & $2.2^{*}$ & $0.29 *$ & $2.6^{*}$ & $5.5^{*}$ & $0.36^{*}$ \\
\hline SY & 3.5 & 3.3 & 0.03 & $1.8^{*}$ & 3.6 & $0.33^{*}$ & 2.5 & $5.5^{*}$ & $0.38^{*}$ \\
\hline \multicolumn{10}{|c|}{ Suprasylvian lesion } \\
\hline BY & 4.2 & 5.8 & 0.16 & $1.1^{*}$ & 5.9 & $0.68^{*}$ & $1.9^{*}$ & 7.3 & $0.53^{*}$ \\
\hline $\mathrm{CE}$ & 2.9 & 2.7 & 0.04 & 2.0 & 2.1 & 0.02 & 2.7 & 2.8 & 0.02 \\
\hline MS & 4.4 & 4.9 & 0.05 & $2.2^{*}$ & 4.0 & $0.29 *$ & 3.8 & $6.0^{*}$ & $0.22 *$ \\
\hline \multicolumn{10}{|c|}{$17 / 18+$ Corpus callosum lesion } \\
\hline $\mathrm{BE}$ & 6.8 & 5.8 & 0.08 & $4.8^{*}$ & $7.7^{*}$ & $0.23^{*}$ & $2.8^{*}$ & 6.8 & $0.42^{*}$ \\
\hline $\mathrm{JX}$ & 4.0 & 4.2 & 0.02 & $2.8^{*}$ & 4.2 & $0.20^{*}$ & 3.1 & 4.2 & $0.15^{*}$ \\
\hline TR & - & - & - & - & - & - & $3.9^{*}$ & $11.9^{*}$ & $0.50^{*}$ \\
\hline \multicolumn{10}{|c|}{ 17/18 Lesion } \\
\hline TR & 5.8 & 6.3 & 0.04 & 5.3 & 6.7 & 0.12 & - & - & - \\
\hline $\mathrm{SE}$ & 3.4 & 3.5 & 0.01 & 3.2 & 3.7 & 0.07 & 3.4 & 3.8 & 0.05 \\
\hline \multicolumn{10}{|c|}{$99 \%$ Confidence intervals } \\
\hline & $0.6-$ & $0.6-1.6$ & $0-0.1$ & & & & & & \\
\hline
\end{tabular}

Time constants for slow phases generated towards (TT) and away (TA) from side of the lesion are shown.

$$
\text { Index of asymmetry }(I)=|| \frac{\mathrm{TT}-\mathrm{TA}}{\mathrm{T} \mathrm{I}+\mathrm{IA}}|| \text {. }
$$

Preoperative measurements were repeated several times to determine $99 \%$ confidence intervals of normal variation, which are listed at the bottom of the table. Postoperative measurements that fall outside of these confidence intervals are marked with an asterisk. Three days postoperatively, time constants became asymmetric in cats with LC, SS, and $17 / 18$ plus corpus callosum lesions. This was primarily due to a decrease in time constants for slow phases directed towards the side of the lesion. Three weeks postoperatively, time constants increased both towards and away from the side of the lesion.

index of asymmetry $(I)$ in the 2 directions. Preoperative measurements were repeated on 3 separate days in order to determine $99 \%$ confidence intervals of normal variation, which are listed at the bottom of the table. Postoperative measurements that fall outside of these confidence intervals are indicated by an asterisk. Three days postoperatively, the time constants of OKAN were reduced for slow phases directed towards the side of the lesion (TT) in cats with LC, SS, and $17 / 18$ plus CC lesions. OKAN time constants were unchanged for slow phases directed away from the side of the lesion $(T A)$ in all cats except for one cat in each of the LC and $17 / 18$ plus CC lesioned groups, where the OKAN time constant was decreased and increased, respectively. By 3 weeks postoperatively, time constants partially recovered for slow phases directed towards the lesion and were often increased over preoperative values for slow phases directed away from the side of the lesion.

\section{Vestibular responses}

Baseline VOR gains in the dark were measured before each VOR gain adaptation training session preoperatively, as well as $3 \mathrm{~d}$ and 3 weeks postoperatively (Table 3 ). Preoperatively, VOR gains to constant velocity steps in head rotation and to sinusoidal rotations at 0.05 and $0.25 \mathrm{~Hz}$ were measured repeatedly to determine the $99 \%$ confidence intervals. Three days postoperatively, VOR gains to velocity steps or $0.05 \mathrm{~Hz}$ sinusoidal rotations were reduced in some cats with $\mathrm{LC}, \mathrm{SS}$, and 17/18 lesions, but there were no significant changes in the index of asymmetry. In one cat with a SS lesion, VOR gain to a velocity step was increased, but the index of asymmetry was not significantly changed. In contrast, for $0.25 \mathrm{~Hz}$ sinusoidal rotations, the index of asymmetry was increased significantly in one cat with a LC lesion (GY) and in one cat with a SS lesion (BY) due to a decrease in the VOR gain for slow phases towards the side of the lesion. By 3 weeks postoperatively, VOR gains bccamc asymmetric in a number of cats with LC, SS, and $17 / 18$ plus $\mathrm{CC}$ lesions to either velocity steps or sinusoidal rotations.

VOR time constants to constant-velocity steps in head rotation did not change significantly during the initial postoperative period in any of the cats (Table 4). By 3 weeks postoperatively, the index of asymmetry became significantly increased in several cats with $\mathrm{LC}$ and $17 / 18$ plus CC lesions. In these cats, time constants were decreased for slow phases towards the side of the lesion, and time constants were increased for slow phases away from the side of the lesion.

An example of an asymmetric VOR gain and time constant in one cat (CO) 3 weeks after a left LC lesion is shown in Figure 5 . To $30 \%$ sec constant-velocity steps in chair rotation, the VOR gain and time constants were 0.40 and 5.0 sec for slow phases directed to the left (towards the side of the lesion) compared with 0.61 and $9.3 \mathrm{sec}$ to the right. The VOR gains to $0.25 \mathrm{~Hz}$ sinusoidal chair rotations were 0.24 to the left and 0.37 to the right and the VOR gains to $0.05 \mathrm{~Hz}$ sinusoidal chair rotations were 0.30 to the left and 0.51 to the right.

When the cats developed significant asymmetries in either VOR gain or time constant, they also developed nystagmus at the termination of each sinusoidal chair rotation (arrow in Fig. 
Table 3. Summary of VOR gains (measured in darkness) to constant velocity steps and to 0.05 and $0.25 \mathrm{~Hz}$ sinusoidal rotations (peak chair velocity $=30 \%$ sec)

\begin{tabular}{|c|c|c|c|c|c|c|c|c|c|}
\hline \multirow[b]{2}{*}{ Cat } & \multicolumn{3}{|c|}{ Pre-op } & \multicolumn{3}{|c|}{3 Days post-op } & \multicolumn{3}{|c|}{3 Weeks post-op } \\
\hline & GT & GA & I & GT & GA & I & GT & GA & I \\
\hline \multicolumn{10}{|c|}{ VOR gains to constant velocity steps } \\
\hline \multicolumn{10}{|c|}{ Large cortical lesion } \\
\hline GY & 0.68 & 0.72 & 0.03 & $0.45^{*}$ & $0.50^{*}$ & 0.05 & $0.49 *$ & 0.77 & $0.22 *$ \\
\hline $\mathrm{CO}$ & 0.59 & 0.63 & 0.03 & 0.55 & 0.64 & 0.08 & $0.40^{*}$ & 0.61 & $0.21^{*}$ \\
\hline SR & 0.55 & 0.50 & 0.05 & 0.55 & 0.50 & 0.05 & $0.34^{*}$ & 0.54 & $0.23 *$ \\
\hline SY & 0.58 & 0.52 & 0.03 & $0.31 *$ & $0.32^{*}$ & 0.02 & 0.43 & 0.45 & 0.02 \\
\hline \multicolumn{10}{|c|}{ Suprasylvian lesion } \\
\hline BY & 0.50 & 0.52 & 0.02 & 0.55 & $0.67^{*}$ & 0.10 & 0.47 & $0.74^{*}$ & $0.22 *$ \\
\hline $\mathrm{CE}$ & 0.77 & 0.73 & 0.03 & 0.72 & 0.76 & 0.03 & 0.68 & 0.67 & 0.00 \\
\hline MS & 0.65 & 0.61 & 0.03 & 0.62 & 0.65 & 0.02 & 0.51 & 0.69 & $0.15^{*}$ \\
\hline \multicolumn{10}{|c|}{$17 / 18+$ Corpus callosum lesion } \\
\hline $\mathrm{BE}$ & 0.81 & 0.86 & 0.03 & 0.76 & 0.80 & 0.02 & 0.69 & 0.79 & 0.07 \\
\hline $\mathrm{JX}$ & 0.76 & 0.81 & 0.03 & 0.52 & 0.55 & 0.03 & 0.66 & 0.68 & 0.01 \\
\hline TR & - & - & - & - & - & - & 0.70 & 0.69 & 0.01 \\
\hline \multicolumn{10}{|c|}{ 17/18 Lesion } \\
\hline TR & 0.91 & 0.95 & 0.02 & 0.81 & 0.88 & 0.04 & - & - & - \\
\hline $\mathrm{SE}$ & 0.74 & 0.84 & 0.06 & 0.80 & 0.83 & 0.02 & 0.54 & 0.51 & 0.03 \\
\hline
\end{tabular}

VOR gains to $0.05 \mathrm{~Hz}$ sinusoidal rotations

Large cortical lesion

\begin{tabular}{|c|c|c|c|c|c|c|c|c|c|}
\hline GY & 0.79 & 0.89 & 0.06 & $0.53^{*}$ & $0.60^{*}$ & 0.06 & $0.47^{*}$ & 0.73 & $0.22 *$ \\
\hline $\mathrm{CO}$ & 0.73 & 0.75 & 0.01 & $0.54^{*}$ & 0.58 & 0.04 & $0.30^{*}$ & $0.51^{*}$ & $0.26^{*}$ \\
\hline SY & 0.54 & 0.56 & 0.02 & $\mathrm{ND}$ & ND & ND & $0.32 *$ & $0.37^{*}$ & 0.07 \\
\hline \multicolumn{10}{|c|}{ Suprasylvian lesion } \\
\hline BY & 0.64 & 0.67 & 0.02 & 0.50 & 0.59 & 0.08 & 0.47 & 0.67 & $0.18^{*}$ \\
\hline CF & 0.93 & 0.87 & 0.03 & $0.64 *$ & $0.62^{*}$ & 0.02 & $0.59^{*}$ & $0.62^{*}$ & 0.02 \\
\hline MS & 0.68 & 0.65 & 0.02 & 0.58 & 0.61 & 0.02 & 0.51 & 0.68 & $0.14^{*}$ \\
\hline \multicolumn{10}{|c|}{$7 / 18+$ Corpus callosum lesion } \\
\hline $\mathrm{BE}$ & 0.87 & 0.97 & 0.05 & 0.87 & 1.01 & 0.08 & $0.61^{*}$ & 1.01 & $0.25^{*}$ \\
\hline TR & - & - & - & - & - & - & $0.60^{*}$ & 0.80 & $0.14^{*}$ \\
\hline \multicolumn{10}{|c|}{ 7/18 Lesion } \\
\hline TR & 0.85 & 0.89 & 0.02 & 0.90 & 0.92 & 0.01 & - & - & - \\
\hline SE & 0.72 & 0.75 & 0.02 & $0.49^{*}$ & $0.51^{*}$ & 0.02 & 0.68 & 0.70 & 0.01 \\
\hline
\end{tabular}

VOR gains to $0.25 \mathrm{~Hz}$ sinusoidal rotations

Large cortical lesion

\begin{tabular}{|c|c|c|c|c|c|c|c|c|c|}
\hline GY & 0.56 & 0.54 & 0.02 & $0.34^{*}$ & 0.48 & $0.17^{*}$ & $0.36^{*}$ & $0.72^{*}$ & $0.33^{*}$ \\
\hline $\mathrm{CO}$ & 0.60 & 0.74 & 0.10 & 0.57 & $0.55^{*}$ & 0.02 & $0.24^{*}$ & $0.37^{*}$ & 0.20 \\
\hline SY & 0.50 & 0.44 & 0.06 & ND & ND & ND & $0.25^{*}$ & 0.32 & 0.12 \\
\hline \multicolumn{10}{|c|}{ uprasylvian lesion } \\
\hline BY & 0.62 & 0.70 & 0.06 & $0.41^{*}$ & 0.62 & $0.21^{*}$ & $0.43^{*}$ & 0.61 & $0.17^{*}$ \\
\hline $\mathrm{CE}$ & 0.74 & 0.72 & 0.01 & $0.55^{*}$ & 0.66 & 0.09 & $0.51^{*}$ & $0.44^{*}$ & 0.07 \\
\hline MS & 0.59 & 0.57 & 0.01 & 0.51 & 0.54 & 0.03 & 0.42 & 0.59 & $0.15 *$ \\
\hline \multicolumn{10}{|c|}{$7 / 18+$ Corpus callosum lesion } \\
\hline $\mathrm{BE}$ & 0.87 & 1.10 & 0.12 & 0.73 & 0.94 & 0.12 & $0.61^{*}$ & 1.01 & $0.25^{*}$ \\
\hline TR & - & - & - & - & - & - & 0.63 & 0.72 & 0.05 \\
\hline \multicolumn{10}{|c|}{ 7/18 Lesion } \\
\hline TR & 0.77 & 0.84 & 0.04 & 0.78 & 0.71 & 0.05 & - & - & - \\
\hline $\mathrm{SE}$ & 0.74 & 0.72 & 0.01 & 0.91 & 0.83 & 0.04 & 0.77 & 0.76 & 0.00 \\
\hline
\end{tabular}

Gains for slow phases generated towards (GT) and away (GA) from the side of the lesion are shown.

$$
\text { Index of asymmetry }(h)=|| \frac{\mathrm{GT}-\mathrm{GA}}{\mathrm{GT}+\mathrm{GA}}|| \text {. }
$$

Postoperative measurements that fall outside of the $99 \%$ confidence intervals measured preoperatively are marked with an asterisk. Three days postoperatively, VOR gains remained symmetric for constant velocity steps and for $0.05 \mathrm{~Hz}$ sinusoidal rotations but were asymmetric to $0.25 \mathrm{~Hz}$ rotations in one cat with a LC lesion (GY) and in one cat with a SS lesion (BY). Three weeks postoperatively, VOR gains became asymmetric in several cats to both constant-velocity steps and sinusoidal rotations. This was due to a decrease in time constants for slow phases towards the side of the lesion and an increase in time constants for slow phases away from the side of the lesion. 
Table 4. Summary of VOR time constants to constant-velocity steps

\begin{tabular}{|c|c|c|c|c|c|c|c|c|c|}
\hline \multirow[b]{2}{*}{ Cat } & \multicolumn{3}{|c|}{ Pre-op } & \multicolumn{3}{|c|}{3 Days post-op } & \multicolumn{3}{|c|}{3 Weeks post-op } \\
\hline & $\mathrm{TT}$ & TA & I & TT & $\mathrm{TA}$ & $\mathrm{I}$ & $\mathrm{TT}$ & TA & $\mathrm{I}$ \\
\hline \multicolumn{10}{|c|}{ Large cortical lesion } \\
\hline GY & 5.7 & 4.7 & 0.10 & 6.2 & 6.1 & 0.01 & $3.2^{*}$ & 5.4 & $0.26^{*}$ \\
\hline $\mathrm{CO}$ & 4.4 & 5.2 & 0.08 & 4.4 & 4.2 & 0.02 & 5.0 & $9.3^{*}$ & $0.30^{*}$ \\
\hline SR & 7.3 & 7.5 & 0.01 & 6.0 & 6.5 & 0.04 & $5.1^{*}$ & 8.9 & $0.27^{*}$ \\
\hline SY & 4.8 & 5.3 & 0.06 & 4.1 & 4.8 & 0.04 & 5.7 & 5.5 & 0.02 \\
\hline \multicolumn{10}{|c|}{ Suprasylvian lesion } \\
\hline BY & 5.3 & 5.2 & 0.01 & 4.5 & 4.2 & 0.03 & 4.2 & 4.0 & 0.02 \\
\hline $\mathrm{CE}$ & 4.6 & 4.4 & 0.02 & 4.6 & 4.0 & 0.07 & 6.2 & 5.8 & 0.03 \\
\hline MS & 4.8 & 4.6 & 0.02 & 4.3 & 4.0 & 0.03 & 4.0 & 4.2 & 0.02 \\
\hline \multicolumn{10}{|c|}{$17 / 18+$ Corpus callosum lesion } \\
\hline $\mathrm{BE}$ & 6.0 & 6.3 & 0.02 & 5.7 & 6.6 & 0.07 & 7.4 & $10.1^{*}$ & $0.15^{*}$ \\
\hline $\mathrm{JX}$ & 4.2 & 4.5 & 0.03 & 4.2 & 4.9 & 0.08 & 4.7 & 5.5 & 0.08 \\
\hline TR & - & - & - & - & - & - & $7.8^{*}$ & $15.0^{*}$ & $0.32^{*}$ \\
\hline \multicolumn{10}{|c|}{ 17/18 Lesion } \\
\hline TR & 9.6 & 10.3 & 0.04 & 9.2 & 11.2 & 0.10 & - & - & - \\
\hline $\mathrm{SE}$ & 5.9 & 5.4 & 0.04 & 4.8 & 4.3 & 0.05 & 5.6 & 4.7 & 0.09 \\
\hline
\end{tabular}

$99 \%$ Confidence intervals

$$
0.6-1.6 \quad 0.6-1.6 \quad 0-0.11
$$

Time constants for slow phases gencratcd towards (TT) and away (T $\Lambda$ ) from the side of the lesion are shown.

$$
\text { Index of asymmetry }(I)=|| \frac{\mathrm{TT}-\mathrm{TA}}{\mathrm{TT}+\mathrm{TA}}|| \text {. }
$$

The postoperative measurements that fall outside of the $99 \%$ confidence intervals measured preoperatively are marked with an asterisk. Time constants remained symmetric $3 \mathrm{~d}$ postoperatively. Three weeks postoperatively, the time constants were asymmetric in 3 cats with $\mathrm{LC}$ lesions and in 2 cats with a $17 / 18$ plus corpus callosum lesion.

6). During sinusoidal rotation in the dark, the VOR response became asymmetric with an increase in peak eye velocity generated away from the side of the lesion (in the direction of the greater VOR gain or time constant). After termination of sinusoidal rotations, nystagmus continued for another $20-60 \mathrm{sec}$. This nystagmus was most pronounced in the cats with the most asymmetric VOR gain or time constants.

\section{Visual-vestibular interactions and VOR gain adaptation}

Visual-vestibular interactions (VOR gains during $\mathrm{X} 2$ and $\mathrm{X} 0$ viewing), and VOR gain adaptation (the degree to which VOR gain in the dark could be changed after prolonged $\mathrm{X} 2$ and $\mathrm{X} 0$ viewing) were tested in all cats except for one with a $\mathrm{LC}$ lesion (SY) and one with a suprasylvian lesion (CE). These measurements from typical cats in each of the 4 lesioned groups are shown in Figure 7 . During the initial postoperative period, visual-vestibular interactions were impaired in cats with $\mathrm{LC}$ and SS lesions (Fig. 7, dotted vertical lines). For example, in cat $\mathrm{CO}$ the VOR gain increased only $40 \%$ during X2 viewing $12 \mathrm{~d}$ postoperatively compared with $74 \%$ preoperatively, and in cat BY VOR gain increased $54 \%$ during $X 2$ viewing 3 d postoperatively compared with $85 \%$ preoperatively. By the second postoperative period, visual-vestibular interactions had recovered in cats with SS lesions but not in cats with LC lesions.

Following the cortical lesions, there was a persistent deficit in the ability to increase VOR gain measured in the dark following prolonged $\mathrm{X} 2$ viewing for slow phases towards the side of the lesion in all cats with LC and SS lesions, and to a certain extent in cats with $17 / 18$ plus corpus callosum lesions (Fig. 7, continuous lines). There was no or little deficit in the ability to decrease VOR gain for slow phases towards the side of the lesion.
VOR gain adaptation sessions also produced progressive asymmetries in VOR gains to the left and right, i.e., VOR gain adaptation resulted in a progressive decrease in VOR gains for slow phases to the left (towards the lesion) but not to the right. This occurred in all cats except for those with a $17 / 18$ lesion. For example, in cat $\mathrm{CO}$ (Fig. $7 A$ ), by the end of the down gain adaptation session $12 \mathrm{~d}$ postoperatively, the VOR gain had been reduced to 0.38 to the right and 0.25 to the left. At the beginning of the VOR gain adaptation session $28 \mathrm{~d}$ postoperatively, this asymmetry in VOR gain had not recovered but was, in fact, even slightly greater $(0.51$ to the right and 0.30 to the left). During the 2 weeks the cat was in its cage between adaptation testing sessions, the VOR gain increased slightly in both directions. By the end of the final adaptation session $28 \mathrm{~d}$ postoperatively, the VOR gain was 0.51 to the right and was reduced to near zero to the left.

\section{Discussion}

We found several ocular motor deficits in cats with unilateral cortical lesions, including a decreased range of retinal-slip velocities that can maximally drive $\mathrm{OKN}$, decreased $\mathrm{OKN}$ gain at all target velocities, asymmetric time constants for both $\mathrm{OKN}$ and VOR, and impaired VOR gain adaptation. Before discussing these deficits, it would be useful to undertake a brief summary of our current understanding of the optokinetic and vestibular pathways in the cat.

Vestibular nystagmus induced by head acceleration continues after termination of the accelerating stimulus. This prolonged nystagmus is believed to be mediated by discharge of the velocity-storage system. The velocity-storage system is believed to consist of a positive-feedback loop created by an efference 
Figure 5. Asymmetric vestibular response in cat $\mathrm{CO} 3$ weeks following a left-sided large cortical lesion. Responses to both 0.25 and $0.05 \mathrm{~Hz}, 30 \%$ sec sinusoidal rotations $(A)$ and $30^{\circ} / \mathrm{sec}$ constant-velocity steps $(B)$ are shown. Eye position $(E)$, eye velocity $(\dot{E})$, and head velocity $(H)$ are shown. The period of zero eye velocity (star in $A$ ) is a calibration mark. VOR gains to 0.25 $\mathrm{Hz}$ sinusoidal chair rotations were 0.24 to the left and 0.37 to the right and gains to $0.05 \mathrm{~Hz}$ sinusoidal chair rotations were 0.30 to the left and 0.51 to the right $(A)$. To $30 \%$ sec constant-velocity steps in chair rotation, VOR gains, and time constants were 0.40 and 5.0 for slow phases directed to the left compared with 0.61 and 9.3 to the right $(B)$.

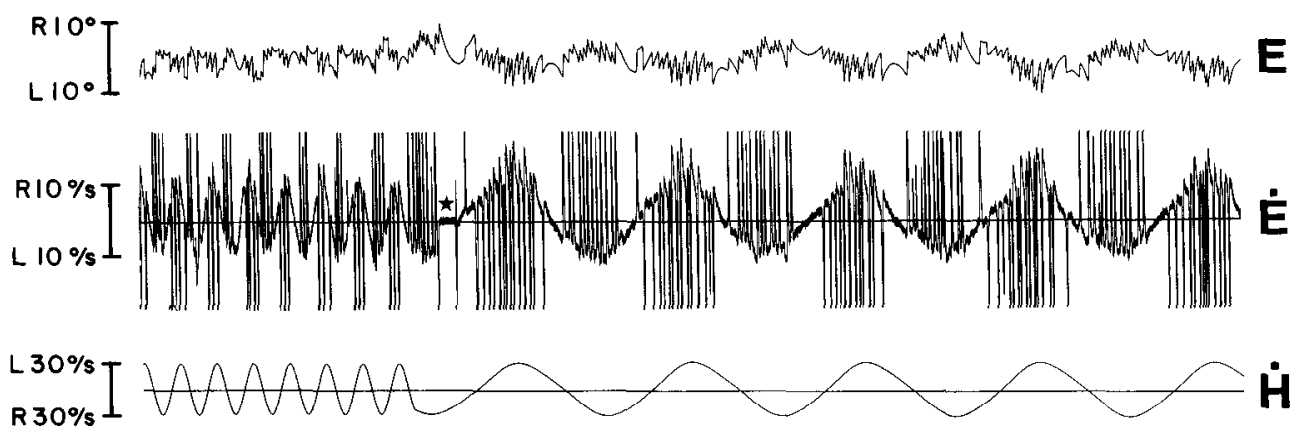

B 205.
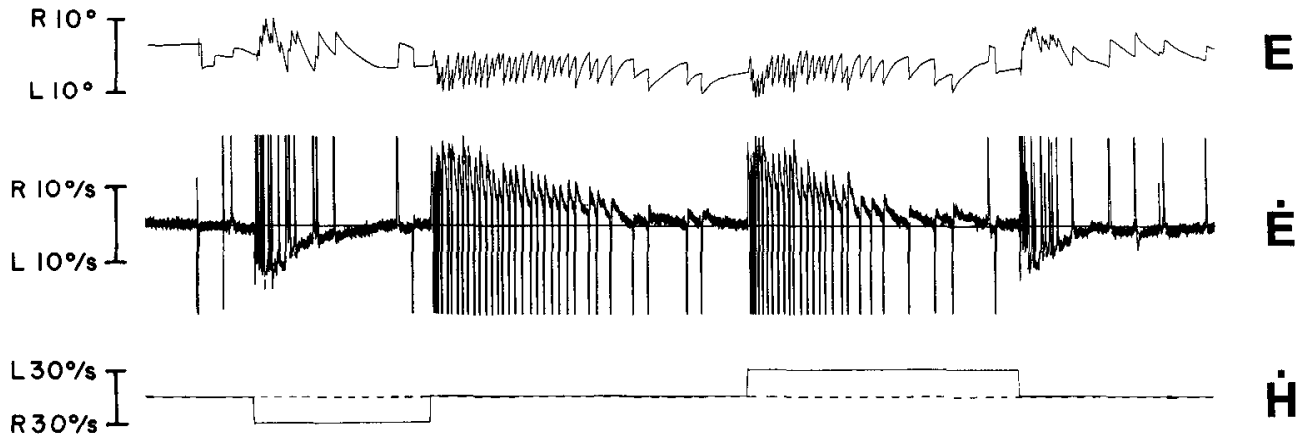

$\dot{H}$ copy of an eye velocity command signal (Robinson, 1981) or by a neural integrator (Raphan and Cohen, 1985).

Slow phases of OKN are driven by 2 components; a quickresponding ("pursuit") component that rapidly increases eye velocity to a proportion of target velocity and a velocity-storage component (shared with the vestibular system) that slowly builds up eye velocity to a steady-state value. When the lights are turned off, slow-phase eye velocity rapidly decreases during the first second due to elimination of the quick-responding component, and then eye velocity slowly decays to zero (OKAN) due to discharge of the velocity-storage component. It is believed that retinal-slip information from the nucleus of the optic tract (NOT) and the accessory optic system (AOS) feeds into the velocity-storage system (Precht, 1982). In the cat the quickresponding component does not play a significant role in the generation of OKN (Maioli and Precht, 1984), and we were unable to make reliable assessments of the effects of cortical lesions on this component.

\section{OKN deficits in cats with unilateral cortical lesions}

Based on examining cats with unilateral lesions, cerebral cortex is believed to enhance the OKN response for ipsilaterally directed slow phases. Cerebral cortex increases the range of retinal-slip velocity to which the OKN system can respond and mediates the OKN response to stimuli moving in the nasal-temporal direction during monocular viewing (Hoffmann, 1982; Strong et al., 1984; Ventre, 1985). The cortical areas primarily involved in OKN are felt to lie within the SS cortex (Ventre, 1985). In this study, we have more precisely determined the critical cortical areas involved in the OKN response. In addition, we have found that cortex not only extends the upper range of retinalslip velocity to which OKN can be elicited, but also increases
Figure 6. Build-up of asymmetric vestibular response to a $30 \% \mathrm{sec}, 0.25$ $\mathrm{Hz}$ sinusoidal chair rotation in cat $\mathrm{CO}$ 8 weeks following a left-sided LC lesion. Eye position $(E)$, eye velocity $(\hat{E})$, and head velocity $(H)$ are shown. During sinusoidal rotation in the dark, the VOR response became asymmetric with an increase in peak eye velocity generated to the right (best seen in the eye velocity trace, $E$ ). When the chair was stopped (arrow), there was a transient jerk nystagmus with slow phases to the right.

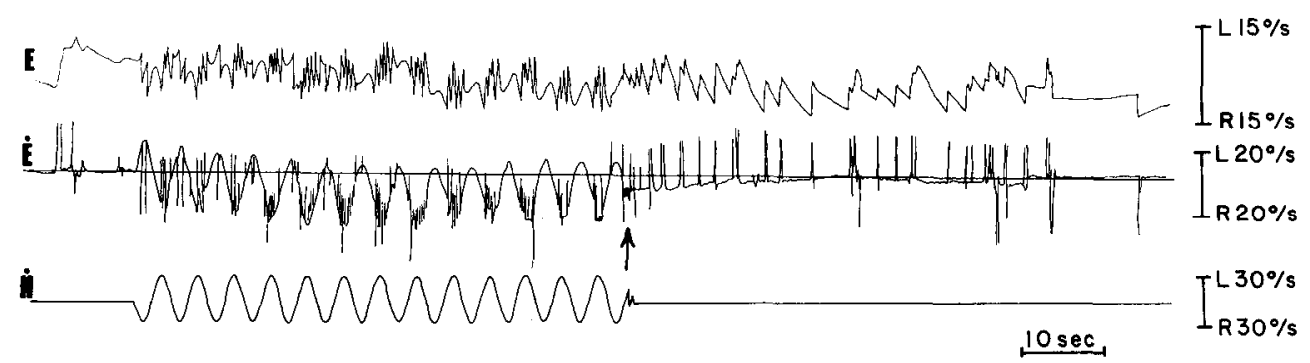


A) Left Large Cortical Lesion (CO) Pre-Op 12 Days Post-op 28 Days Post-Op

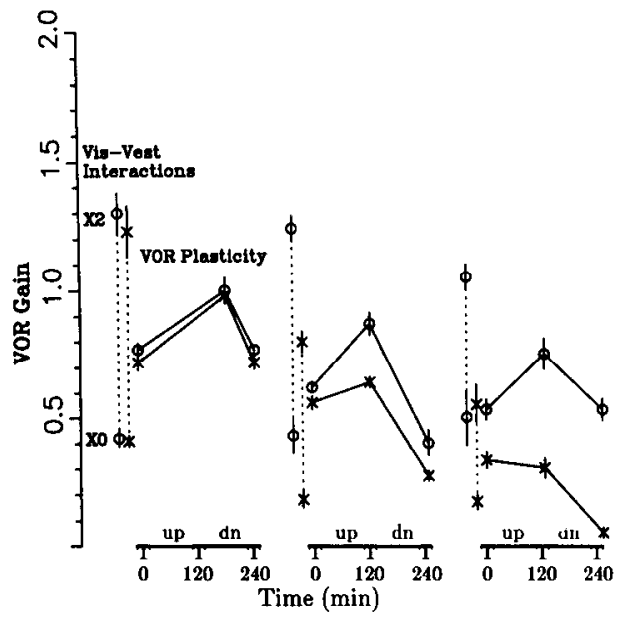

C) Left 17/18 + Corpus Callosum Lesion (BE) Pre-Op 8 Daya Post-Op 43 Daya Post-Op

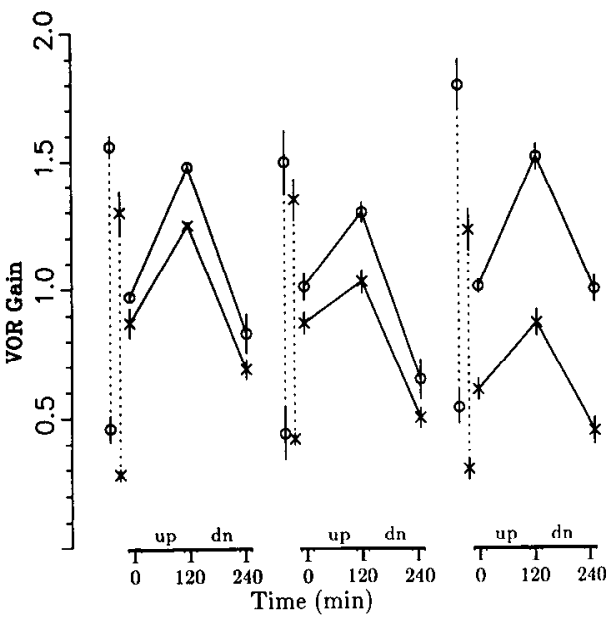

B) Left Suprasylvian Lesion (BY) Pre-Op 3 Days Post-Op 19 Days Post-Op

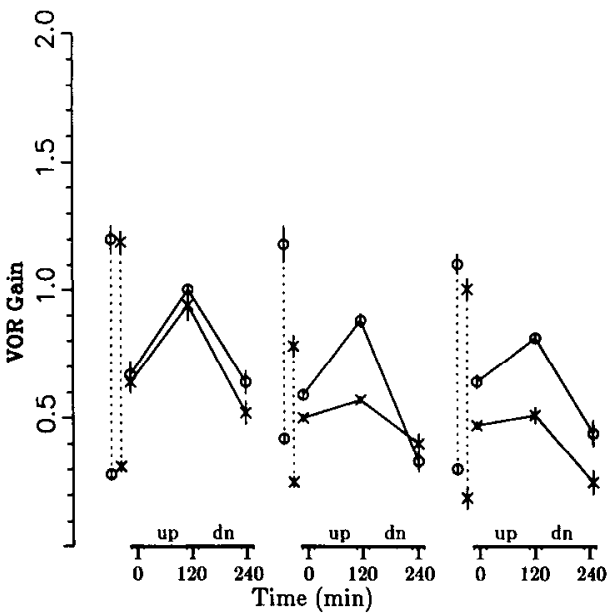

D) Left 17/16 Lesion (SE) Pre-Op 2 Days Post-op 41 Daya Post-Op

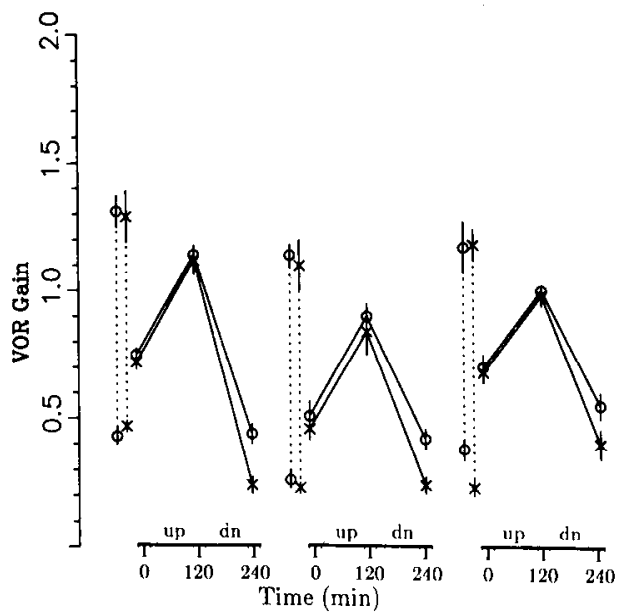

Figure 7. Graphs of VOR gain adaptation and visual-vestibular interactions in one cat from each lesion group for $30 \% \mathrm{sec}, 0.05 \mathrm{~Hz}$ sinusoidal rotations. Open circles and crosses indicate VOR gains for slow phases to the right and left, respectively. Adaptation was measured preoperatively and twice postoperatively. During each day adaptation was assessed, visual-vestibular interactions were first determined, i.e., the ability to modulate VOR gain during $\mathrm{X} 2$ and $\mathrm{X} 0$ viewing (illustrated as vertical dotted lines). VOR gain adaptation was then measured following prolonged $\mathrm{X} 2$ viewing $(u p)$ and $\mathrm{X} 0$ viewing (down). In cat $\mathrm{CO}, \mathrm{X} 2$ viewing lasted for $180 \mathrm{~min}$ and $\mathrm{X} 0$ viewing lasted for $60 \mathrm{~min}(A)$. In all other cats, $\mathrm{X} 2$ viewing and $\mathrm{X} 0$ viewing each lasted for $120 \mathrm{~min}$. Values interconnected by continuous lines are means for 6-10 measurements of VOR responses in the dark after each prolonged $\mathrm{X} 2$ or $\mathrm{X} 0$ viewing session. Ability to modulate VOR gain during visual-vestibular interactions was initially impaired in cats with LC and SS lesions $(A$ and $B)$. The relative increase in gain for slow phases to the left was less during $\mathrm{X} 2$ viewing compared with preoperative values. By the second postoperative period, visual-vestibular interactions recovered in cats with SS lesions. The ability to increase VOR gain measured in the dark following VOR gain adaptation towards the side of the lesion was impaired in cats with LC, SS, and to a certain extent in cats with $17 / 18$ plus corpus callosum lesions $(A-C)$. Postoperatively, VOR gain adaptation sessions also resulted in a progressive asymmetry in VOR gains in all cats except for those with $17 / 18$ lesions.
OKN gain at all target velocities and influences the OKAN time constant.

\section{Retinal-slip velocity}

Areas PMLS, VLS, $21 \mathrm{a}$, and $21 \mathrm{~b}$ appear to be the critical region responsible for increasing the range of retinal-slip velocity to which the OKN system can respond. Metabolic activity is significantly increased in these areas in cats generating OKN (Herdman et al., 1989). Unilateral ablation of these areas initially reduces the range of retinal-slip velocity that can drive OKN to the same extent as a large unilateral cortical lesion involving all known visually responsive cortex.

The properties of neurons in the SS region are well suited for processing retinal-slip velocity for OKN. The majority of cells in PMLS are velocity tuned with peak firing rates occurring for target velocities ranging between $1^{\circ}$ and $100 \%$ sec (Spear and Baumann, 1975; Camarda and Rizzolatti, 1976). In addition, areas PMLS, VLS. $21 \mathrm{a}$, and $21 \mathrm{~b}$ contain an extensive representation of either the area centralis or the horizontal meridian (Tusa et al., 1981), which is the region of the visual field that elicits OKN optimally (Hoffmann and Schoppmann, 1981). Finally, areas PMLS, $21 \mathrm{a}$, and $21 \mathrm{~b}$ have a strong projection to the AOS (Berson and Graybiel, 1980; Marcotte and Updyke, 1982).

$\Lambda$ reas PMLS, VLS, $21 \mathrm{a}$, and $21 \mathrm{~b}$ receive visual input from a number of cortical and subcortical regions, including areas 17 and 18, pulvinar and lateral posterior nuclei (see Rosenquist, 1985). Based on our study we believe that for the generation of OKN the SS region primarily depends on retinal-slip velocity information from areas 17 and 18 . Unilateral ablation of areas 17 and 18 has no affect on OKN, but when this lesion is paired with section of the corpus callosum, the maximum effective retinal-slip velocity for slow phases ipsilateral to the $17 / 18$ lesion is significantly reduced. Montarolo et al. (1981) have also reported that $\mathrm{OKN}$ saturates at a lower target velocity following bilateral 17-19 lesions. Thus, SS cortex in one hemisphere appears to be able to use retinal-slip information from areas 17 and 18 in the same hemisphere or from the contralateral hemisphere via the corpus callosum. Ablation of areas 17 and 18 in the cat also results in a persistent reduction in the proportion 
Figure 8. Control systems model of the horizontal vestibular-optokinetic system of the cat. $H$, head velocity; $H_{c}$, canal estimate of head velocity; $\dot{H}_{v}$, velocity storage estimate of head velocity; $H_{0}$, combined estimate of head velocity; $G_{R}$ and $G_{L}$, variable gain elements for eye velocity commands to the right and left; $K_{R}$ and $K_{r}$, efference copy elements for eye-velocity commands to the right and left; CVSE, central velocity storage element containing a neural integrator with a time constant of $T_{\mathrm{o}} ; E^{\prime}$, eye velocity command signal; $P L A N T$, oculomotor plant; $\dot{E}$, slow-phase eye velocity; $G$, gaze velocity; $r$, total visual velocity command; $r_{R}$ and $r_{L}$, visual velocity commands from the right and left eyes; $N_{R}$ and $N_{L}$, retinal-slip nonlinearities for right and left eyes; $S_{R}$ and $S_{L}$, patches over the right and left eyes; $\dot{e}$, retinal-slip velocity; $S$, switch representing illumination; $W^{\prime}$, velocity of the seen world; $\dot{W}$, velocity of visual surroundings; $M-1$, effective magnification of the seen world. See text for specifics.

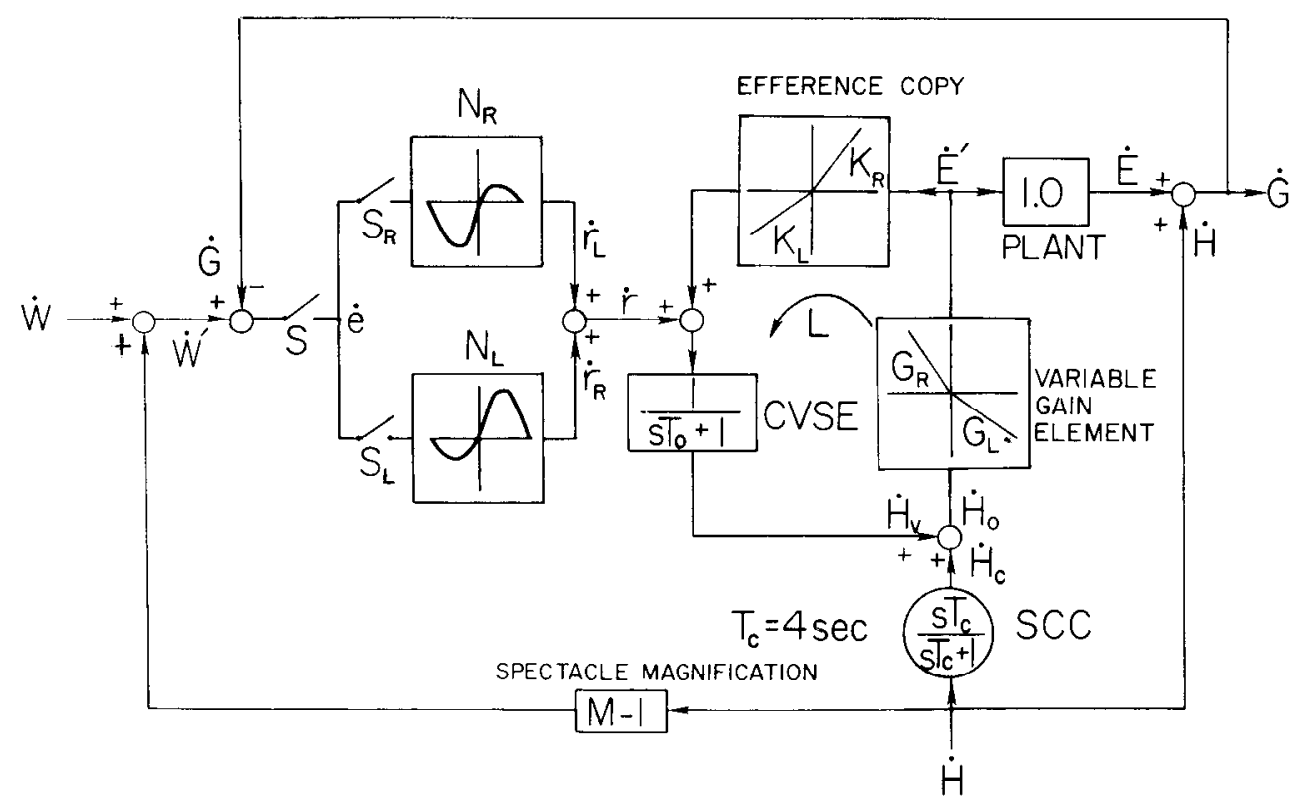

of directionally selective cells in PMLS from $81 \%$ in the normal cat to $7.5 \%$ in the lesioned cat (Spear and Baumann, 1979), although the effect of such a lesion on the processing of retinalslip velocity information in PMLS is unknown.

Within 3 weeks of a SS lesion, we found that optokinetic responses to high retinal-slip velocities recovered to near preoperative values. In contrast, we found no significant recovery in OKN during the 6 week follow-up period in cats with LC or $17 / 18$ plus CC lesions. Recovery within 3 weeks in cats with the SS lesions may occur because the lesions were incomplete or because other areas, such as areas 17 and 18, can eventually provide high retinal-slip velocity information to subcortical regions involved in OKN. The NOT does receive a sparse projection from areas 17 and 18 (Kawamura et al., 1974; Updyke, 1977; Schoppmann, 1981). The fact that OKN did not significantly recover in cats with the $17 / 18$ plus $\mathrm{CC}$ lesions within 6 weeks suggests that, within this time frame, SS cortex cannot use additional afferent sources to process high retinal-slip velocities. Strong et al. (1984) have also reported some recovery of OKN in cats with large unilateral cortical lesions, but they found a progressive decrease in $\mathrm{OKN}$ in the opposite direction as optokinetic responses improved towards the side of the lesion. We did not find a decrease in the contralaterally directed optokinetic responses. This discrepancy may be due to the location of the lesion. This is further discussed below (OKN generated away from the side of the lesion).

\section{OKN gain and OKAN time constant}

In the cats with LC, SS, and 17/18 plus CC lesions, there was a reduction in $\mathrm{OKN}$ gain at all stimulus velocities measured for slow phases directed ipsilateral to the lesion. Decreased OKN gain at all stimulus velocities has also been reported in cats with large unilateral SS lesions (areas 5, 7, 21a, AMLS, PMLS, and PLLS) with or without involvement of areas 17-19 (Hoffmann, 1982; Ventre, 1985). This decrease in OKN gain could be accounted for by 2 different neural mechanisms. First, it may be due to an overall decrease in neural activity in NOT/AOS to moving stimuli. In the normal cat, higher retinal-slip velocities result in greater neural activity in the NOT/AOS, which, in turn, correlates with higher slow-phase eye velocity (Hoffmann and Distler, 1986). If after a cortical lesion, retinal-slip velocity now resulted in reduced neural activity in NOT/AOS compared to normal, then decreased OKN gain would be expected to occur. Unilateral cortical lesions in the cat do reduce the proportion of neurons in AOS that respond to high stimulus velocities (Grasse et al., 1984), although it is not known whether the neuronal response in AOS is reduced at low retinal-slip velocities. Alternatively, the decrease in OKN gain following a unilateral cortical lesion may be due to a decrease in gain within the velocity-storage mechanism. Computer simulations of the OKN response in a combined VOR-OKN mathematical model are shown in Figure 9 of the Appendix. In these simulations we used the data from cat GY, who developed a significant reduction in gain in the velocity storage system immediately after a LC lesion. When the gain of the velocity-storage system is reduced in the model, the maximum OKN gain and peak slowphase eye velocity are appropriately reduced. Changes in the velocity-storage system do not reduce the range of retinal-slip velocity that can drive $\mathrm{OKN}$, though, which suggests that the $\mathrm{OKN}$ deficit we observed following this lesion is not totally due to changes in the velocity-storage system, but rather must be also due to changes in the retinal-slip nonlinearity within the $\mathrm{OKN}$ system. We can only speculate how cerebral cortical lesions could cause changes in the velocity-storage system. The velocity-storage system is thought to involve the nucleus reticularis tegmenti pontis (NRTP), prepositus hypoglossi nucleus, vestibular nucleus, and their interconnections (Precht, 1982; Lannou et al., 1984; Ie Taillanter and Lannou, 1988). It is possible that the neural activity within NRTP is remotely affected by cortical lesions. In the cat, NRTP receives extensive projections from frontal and parietal cortex and possibly minor projections from occipital cortex (Brodal and Brodal, 1971). 
OKN generated away from the side of the lesion

During binocular viewing, optokinetic slow phases directed away from the side of the lesion were unchanged from preoperative values in all cats. During monocular viewing, the effect of the lesion varicd depending on whether SS cortex was involved. In the cats in which SS cortex was spared, slow-phase eye velocity was either unchanged ( $17 / 18$ lesions) or lower (17/18 plus corpus callosum lesions) than preoperative values. In the animals where SS cortex was lesioned, slow-phase eye velocity was usually increased. This change in the optokinetic response was primarily due to a change in maximum gain, and not a change in the range of retinal-slip velocities that could drive $\mathrm{OKN}$.

Optokinetic slow phases are thought to be generated by excitation of the NOT/AOS complex contralateral to the direction of the slow phases and inhibition of the NOT/AOS complex on the other side (Hoffmann, 1982). Thus, a change in the optokinetic response directed contralateral to the side of the lesion may be due to a change in inhibition of NOT/AOS on the side of the lesion. Alternatively, a change in the optokinetic response may be due to a change in excitatory sensitivity of the NOT/ $\Lambda$ OS complex contralateral to the side of the lesion. Contralateral projections to the NOT/AOS complex from SS cortex have been found in the cat (Berman and Payne, 1982; Baleydier et al., 1983), but it is unknown whether these projections are inhibitory or excitatory.

\section{Vestibular responses}

The ability to alter VOR gain in the dark following prolonged $\mathrm{X} 2$ and $\mathrm{X} 0$ viewing sessions (VOR gain adaptation) was persistently impaired in cats with LC, SS, and to a certain extent in cats with $17 / 18$ plus corpus callosum lesions. This deficit primarily affected gain enhancement and not gain reduction. This selective deficit in gain enhancement has also been found in monkeys following bilateral occipital lobectomies (Fetter et al., 1988), and it may be due to gain enhancement being a more demanding process than gain reduction. In normal monkeys, the rate of gain enhancement is much slower (up to 5-fold) than that of gain reduction (Miles and Eighmy, 1980).

Normal VOR gain adaptation depends on a combination of retinal-slip velocity signals and either eye velocity (efference copy) or head velocity signals to compute crrors in the VOR, based on studies in rabbits and monkeys (Collewijn and Grootendorst, 1979; Lisberger et al., 1984). Whether these same signals are used in VOR gain adaptation in the cat is unknown. All cats in our study who had a deficit in VOR gain adaptation either had an impaired OKN response to high retinal-slip velocities or a decrease in their efference copy of eye velocity as reflected by the decrease in their OKAN time constant.

During the initial postoperative period, VOR gains to constant velocity steps were reduced in both directions in 2 cats with LC lesions and significantly increased away from the side of the lesion in 1 cat with a SS lesion, but the index of asymmetry did not reach statistical significance in any of the cats. Following prolonged $\mathrm{X} 2$ and $\mathrm{X} 0$ viewing, though, VOR gains to constant velocity steps became significantly asymmetric in all cats with LC and SS lesions, and they remained asymmetric for several weeks between VOR gain adaptation sessions. In addition, VOR time constants became significantly asymmetric in many of these cats. VOR gains and time constants did not significantly change in the 2 cats that did not undergo VOR gain adaptation, including one with a LC lesion (SY) and one with a SS lesion (CE).
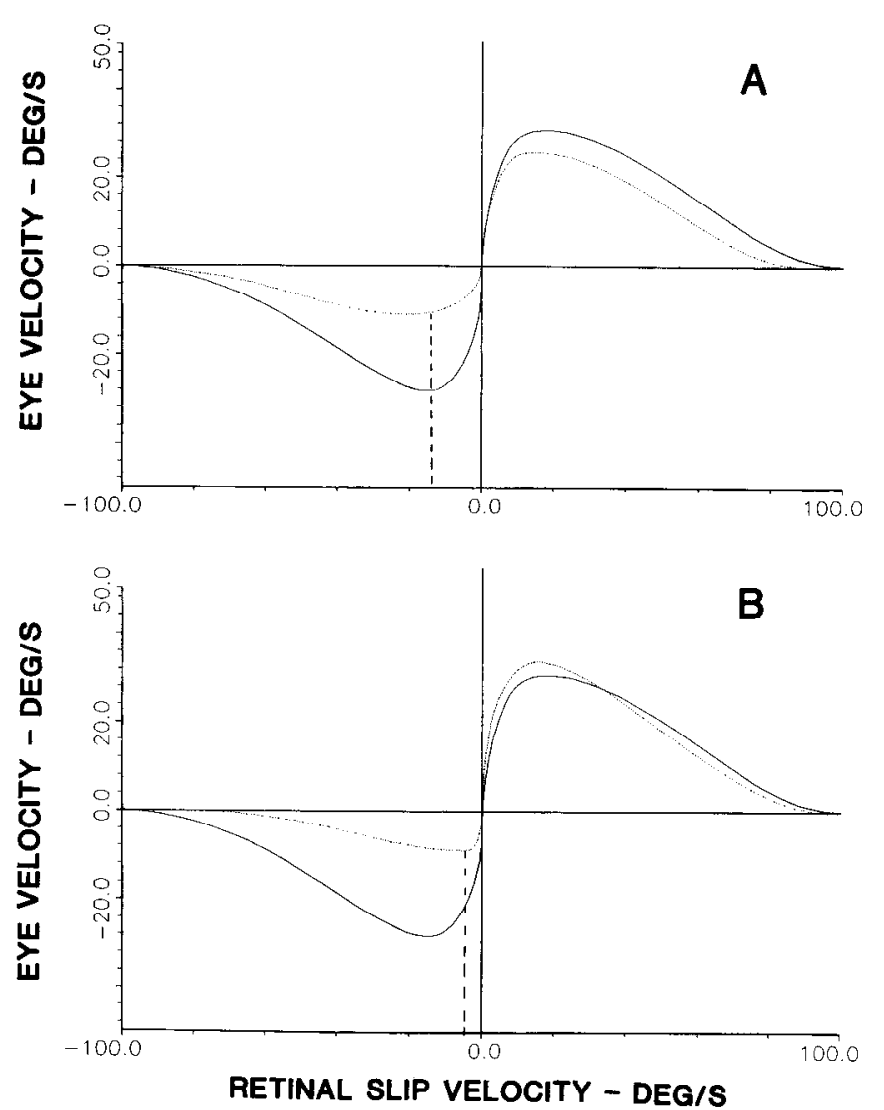

Figure 9. Computer simulations of the steady-state slow-phase eye velocity as a function of retinal-slip velocity during binocular optokinetic stimulation in cat GY before (solid line) and $3 \mathrm{~d}$ after a left-sided large cortical lesion (dotted line). $A$, Effect of changing the variable gain and efference copy elements of the velocity-storage system. $B$, Additional effects of changing the retinal-slip nonlinearity feeding into the velocity-storage system. Eye velocities to the left are negative and eye velocities to the right are positive. For the preoperative OKN simulation, the following parameters were used: $G_{L}$ and $G_{R}=0.7$ (based on the observed preoperative VOR gains to velocity steps); $K_{L}$ and $K_{R}=$ 0.6 (based on equation 2, see text); $T_{\mathrm{o}}=3.0 \mathrm{sec}$ (see text); the retinalslip nonlinearity was based on the measured OKN response. For the postoperative $\mathrm{OKN}$ simulation in $A$ the following parameters were used: $G_{L}=0.45, G_{R}=0.5, K_{L}=0, K_{R}=1.0, T_{0}=1.0 \mathrm{sec}$ (based on the observed postoperative VOR gains, and VOR and OKAN time constants); the retinal-slip nonlinearity was unchanged from preoperative values. Changes in these parameters reduced peak eye velocity from $29^{\circ}$ to $11 \% \mathrm{sec}$ to the left and reduced maximum gain from 0.80 to 0.62 . In order to achicve a best fit to the actual postoperative $\mathrm{OKN}$ response (as shown in $B$ ), a change in the retinal-slip nonlinearity feeding into the velocity-storage system had to be made. In this simulation, the retinalslip velocity at peak eye velocity (indicated by vertical dashed line) had to be decreased and the OKN gain away from the side of the lesion had to be increased.

We believe the development of VOR gain and time constant asymmetries in the cats that underwent VOR adaptation is due to the cat's ability to decrease the gain of the velocity-storage system towards and away from the side of the lesion, and their inability to bring the gain back up again towards the lesioned side. Computer simulations of the VOR response in which the elements within the velocity-storage system were set to the observed values in cat GY several days following VOR gain adaptation are shown in Figure 10 of the Appendix. We can easily simulate asymmetries in the VOR gain and time constant that closely match the observed values in this cat. If the elements within the velocity-storage system are made asymmetric enough 
A

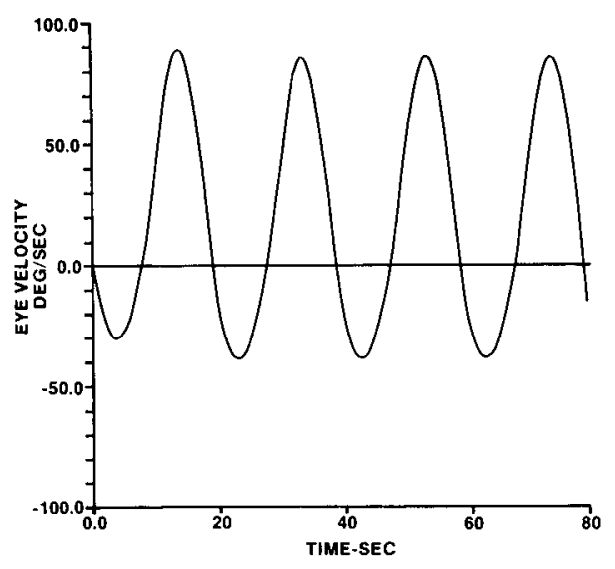

Figure 10. Simulation of VOR in cat GY several days after VOR gain adaptation. This cat had a large cortical lesion in the left hemisphere 3 weeks previously. Stimuli are $0.05 \mathrm{~Hz}$ sinusoids $(A)$ and steps $(B)$ of head velocity having peak velocities of $100 \% \mathrm{sec}$. These simulations were done by setting the variable gain and the efference copy elements of the velocity-storage system to the observed postoperative values: $G_{L}$ $=0.49 ; G_{R}=0.77 ; K_{L}=0.33 ; K_{R}=0.6$; $T_{0}=3.0$ sec. Quick phases were not simulated. The predicted sinusoidal VOR gain in the simulation is 0.38 to the left (negative) and 0.88 to the right (positive), which qualitatively agrees with the observed $0.05 \mathrm{~Hz}$ sinusoidal gain of 0.47 to the left and 0.73 to the right (Table 3 ). The predicted VOR step gain in the simulation is 0.49 to the left and 0.77 to the right, which matches the observed values (Table 3 ), and the predicted step VOR time constant in the simulation is $4 \mathrm{sec}$ to the left and 6 $\mathrm{sec}$ to the right, which compares favorably to the observed values of $3.2 \mathrm{sec}$ to the left and $5.4 \mathrm{sec}$ to the right (Table 4). See text for explanation of how cortical lesions can produce these changes in the variable gain and efference copy elements of the model.

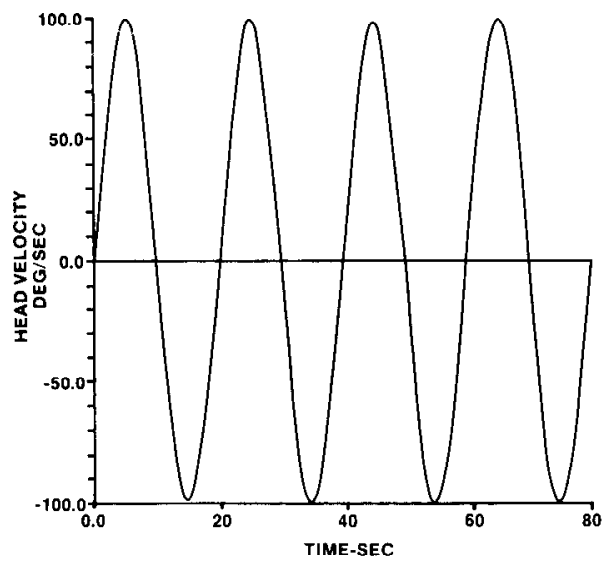

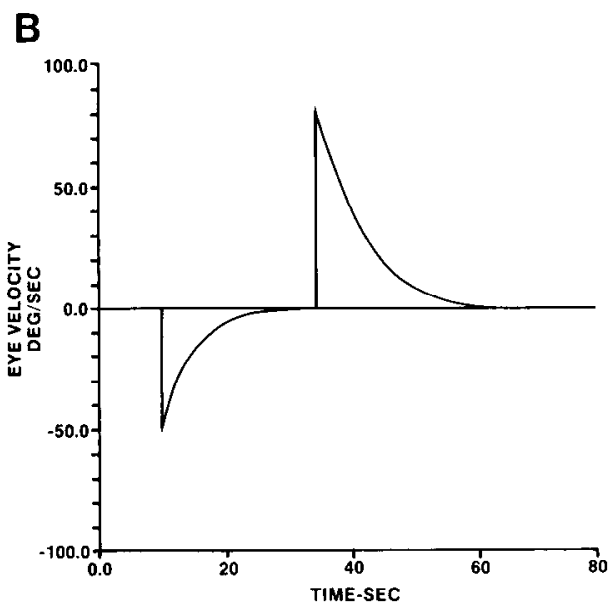

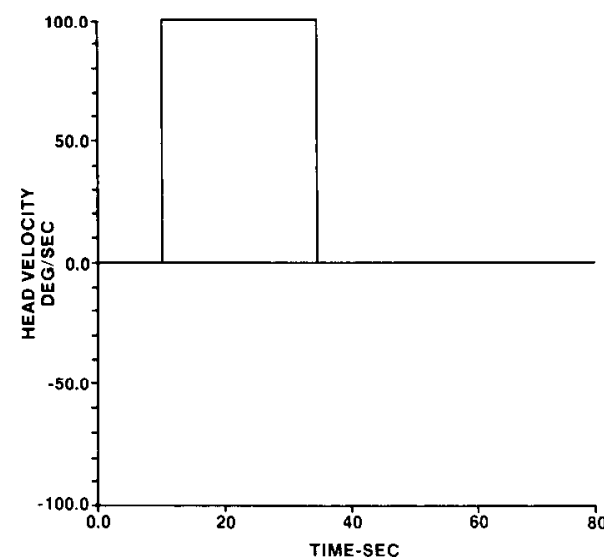

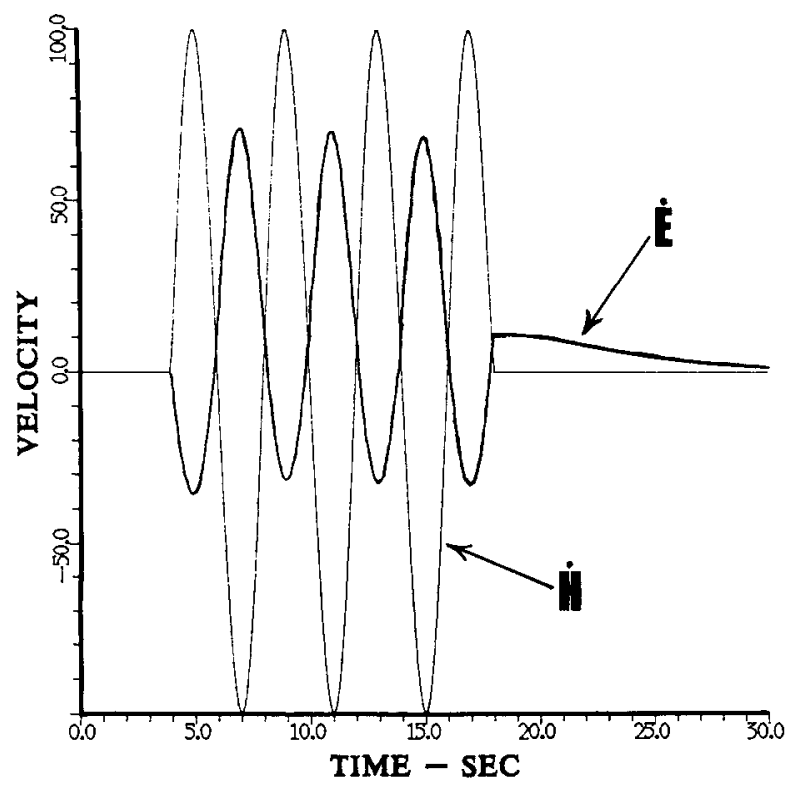

Figure 11. Simulation of VOR gain bias to the right and transient nystagmus after termination of head rotation in cat $\mathrm{CO} 8$ weeks after a left-sided large cortical lesion. The light line represents head velocity $(I)$, and the heavy line represents eye velocity $(E)$. In this simulation the variable gain and efference copy elements were set to the postopcrative obscrved valucs: $G_{L}=0.40 ; G_{R}=0.55 ; K_{L}=0.2 ; K_{R}=0.9 ; T_{0}$ $=1.2 \mathrm{sec}$. Positive eye velocity represents slow phases directed to the right. At the termination of a $12 \mathrm{sec}$ period of sinusoidal head rotation at $0.25 \mathrm{~Hz}$ in darkness, there was a nystagmus with slow phases directed to the right that gradually declined to zero (quick phases were not simulated). in the model, then sinusoidal vestibular stimulation will produce a progressive bias in VOR gain and transient nystagmus after termination of the stimulus (Fig. 11 of Appendix). We observed this in several cats with LC and SS lesions after extensive VOR gain adaptation sessions. We believe this bias in VOR gain occurs because the central eye velocity-storage mechanism does not have time to completely discharge to zero velocity before beginning the next cycle of rotation.

\section{Appendix}

The many complex effects of cortical lesions on the VOR and OKN systems of the cat can be usefully conceptualized in terms of changes in a few components of a mathematical model of the VOR-OKN system (Fig. 8). We used a modification of Robinson's (1977) model in which the individual clements within the positive-fecdback, velocity-storage loop (L) could be adjusted for eye velocity to the right and left. The rationale of this model has been discussed in previous papers (Robinson, 1977, 1981; Demer and Robinson, 1983). In the model, the vestibuloocular reflex begins with transduction of head velocity $(\dot{\mathrm{H}})$ by the semicircular canals (SCC) to produce a neurally encoded head velocity signal $\left(H_{c}\right)$. This transduction is designated by a transfer function shown in Laplace transform terminology, where $T_{c}$ is the cupula time constant and $s$ is the Laplace complex variable. $\dot{H}_{c}$ is then added to the neural estimate of head velocity determined by the visual system $\left(\dot{H}_{v}\right)$. The combination of $\dot{H}_{c}$ and $\dot{H}_{v}$ results in the best visual-vestibular estimate of head velocity $\left(\dot{H}_{\mathrm{v}}\right) . \dot{H}_{\mathrm{v}}$ is multiplied by the variable gain element (the slopes of the nonlinearity are $G_{R}$ and $G_{L}$ for eye velocity commands to the right and left) to produce an eye velocity command $\left(\dot{E}^{\prime}\right)$. The eye velocity command signal drives the oculomotor PLANT (assumed to have unity transfer function) to produce slow-phase eye velocity $(\dot{E})$. The eye velocity command is also multiplied by an efference copy element (the slopes of the nonlinearity are $K_{R}$ and $K_{L}$ for eye velocity commands to the right and left) and the resulting product enters the central velocity storage element (CVSE), consisting of a leaky integrator 
with time constant $T_{0}$. The sum of eye velocity in the head $(\dot{E})$ and head velocity in space $(\dot{H})$ results in gaze velocity in space $(\dot{G})$. The VOR normally acts to reduce gaze velocity to zero to eliminate retinalimage slip; however, if the visual world is artificially moved in some fixed relationship to head velocity (as by a servomechanism driving an optokinetic drum as in our study to induce $\mathrm{X} 2$ or $\mathrm{X} 0$ viewing), retinalslip will occur. The parameter $M$ represents the effective magnification of the servomechanism. Thus, when an observer is horizontally rotated in an optokinetic drum stationary in space, $M=1$. If the drum moves in opposite phase with the observer ( $2 \mathrm{X}$ viewing), $M=2$, and if the drum moves in-phase with the observer (X0 viewing), $M=0$. The product of head velocity $(H)$ and the magnification factor $(M-1)$ is added to the velocity of the visual surroundings $(W)$ to produce a variable representing the velocity of the seen world $\left(W^{\prime}\right)$. Gaze velocity in space $(G)$ is subtracted from $\dot{W}^{\prime}$ to produce retinal-slip velocity $(e)$. When the lights are on, switch $S$ is closed, allowing visual input into the optokinetic system. Opening switches $S_{L}$ or $S_{R}$ represents the effect of patching the left or right eye. With $S_{L}$ and $S_{K}$ closed, both eyes are open and retinal-slip velocity reaches the OKN nonlinearities mediated by the left and right eye $\left(N_{L}\right.$ and $\left.N_{R}\right)$. The curves $N_{L}$ and $N_{R}$ are asymmetric to reflect the nasaotemporal differences observed for monocular OKN. The outputs of the nonlinearities are added to produce a velocity command signal $(r)$, which enters the positive-feedback, velocity storage loop (L) through which they drive eye movements.

The parameters of the model were selected as follows: The vestibular canal time constant $\left(T_{c}\right)$ was set to $4.0 \mathrm{sec}$ based on VIIIth nerve recordings in the cat (Melvill-Jones and Milsum, 1971); the time constant of the central velocity storage element $\left(T_{0}\right)$ was set from 1 to $4 \mathrm{sec}$ based on the difference between the VOR and OKAN time constants (Demer and Robinson, 1983); the variable gain elements $\left(G_{R}\right.$ and $\left.G_{L}\right)$ were based on the gain of the vestibular response to constant-velocity steps; the efference copy parameters were based on equation 2 (Demer, 1981; $T_{\text {OKAN }}$ is the OKAN time constant, $T_{\mathrm{o}}$ is the time constant of the central velocity storage element, $G$ is the variable gain element, and $K$ is the efference copy element):

$$
T_{\text {OKAN }}=T_{\mathrm{o}} /(1-G K) .
$$

The OKN nonlinearities $\left(N_{R}\right.$ and $\left.N_{L}\right)$ were based on the observed steadystate slow-phase eye velocity at several different retinal-slip velocities. Optokinetic and vestibular simulations were implemented on a digital computer using the ASP modeling package, created by H.M. Goldstein and L.M. Optican. Computer simulations of the OKN and VOR responses for cats GY and $\mathrm{CO}$ are shown in Figures 9-11.

\section{References}

Baleydier, C., M. Kahungu, and F. Maugiere (1983) A crossed corticotectal projection from the lateral suprasylvian area in the cat. $\mathrm{J}$. Comp. Neurol. 214: 344-351.

Berman, N., and B. R. Payne (1982) Contralateral corticofugal projections from the lateral suprasylvian and ectosylvian gyri in the cat. Exp. Brain Res. 47: 234-238.

Berson, D. M., and A. M. Graybiel (1980) Some cortical and subcortical fiber projections to the accessory optic nuclei in the cat. Neuroscience 5: 2203-2217.

Brodal, A., and P. Brodal (1971) The organization of the nucleus reticularis tegmenti pontis in the cat in the light of experimental anatomical studies of the cerebral cortical afferents. Exp. Brain Res. 13: $90-110$.

Camarda, R., and G. Rizzolatti (1976) Visual receptive fields in the lateral suprasylvian area (Clare-Bishop area) of the cat. Brain Res. 101: $427-443$.

Collewijn, H., and A. F. Grootendorst (1979) Adaptation of optokinetic and vestibulo-ocular reflexes to modified visual input in the rabbit. In Progress in Brain Research, R. Granit and O. Pompeiano, eds., pp. 772-781, Elsevier, Amsterdam.

Demer, J. L. (1981) The variable gain element of the vestibulo-ocular reflex is common to the optokinetic component of the cat. Brain Res. 220.1-13.

Demer, J. L., and D. A. Robinson (1983) Different time constants for optokinetic and vestibular nystagmus with a single velocity-storage element. Brain Res. 276: 173-177.

Demer, J. L., R. J. Tusa, and S. J. Herdman (1983) Unilateral cerebral cortical ablations impair vestibulo-ocular reflex (VOR) gain and plasticity in the cat. Soc. Neurosci. Abstr. 9: 868.

Fetter, M., D. S. Zee, and L. R. Proctor (1988) Effect of lack of vision and of occipital lobectomy upon recovery from unilateral labyrinthectomy in rhesus monkey. J. Neurophysiol. 59: 394-407.

Grasse, K. L., M. S. Cynader, and R. M. Douglas (1984) Alterations in response properties in the lateral and dorsal tcrminal nuclei of the cat accessory optic component following visual cortex lesions. Exp. Brain Res. 55: 69-80.

Harris, L. R., and M. Cynader (1981) Modification of the balance and gain of the vestibulo-ocular reflex in the cat. Exp. Brain Res. 44: 5770.

Herdman, S. J., R. J. Tusa, and C. B. Smith (1989) Cortical areas involved in horizontal $\mathrm{OKN}$ in cats: Metabolic activity. J. Neurosci. 9: $1150-1162$.

Hoffmann, K.-P. (1982) Cortical versus subcortical contributions to the optokinetic reflex in the cat. In Functional Basis of Ocular Motility Disorders, G. Lennerstrand, D. S. Zee, and E. L. Keller, eds., pp. 303310, Pergamon, Oxford, UK.

Hoffmann, K.-P., and C. Distler (1986) The role of direction selective cells in the nuclcus of the optic tract of cat and monkey during optokinetic nystagmus. In Adaptive Processes in Visual and Oculomotor Components, E. L. Keller and D. S. Zee, eds., pp. 261-274, Pergamon, Oxford, UK.

Hoffmann, K.-P., and A. Schoppmann (1981) A quantitative analysis of the direction-specific response of neurons in the cat's nucleus of the optic tract. Exp. Brain Res. 42: 146-157.

Kawamura, S., J. M. Sprague, and K. Nimi (1974) Corticofugal projections from the visual cortices to the thalamus, pretectum and superior colliculus in the cat. J. Comp. Neurol. 158: 339-362.

Lannou, J., L. Cazin, W. Precht, and M. Le Taillanter (1984) Responses of prepositus hypoglossi neurons to optokinetic and vestibular stimulations in the rat. Brain Res. 301: 39-45.

Le Taillanter, M., and J. Lannou (1988) Responses of nucleus reticularis tegmenti pontis ncurons to vestibular stimulation in the rat. Exp. Brain Res. 69: 417-423.

Lisberger, S. G., F. A. Miles, and D. S. Zee (1984) Signals used to compute errors in monkey vestibuloocular reflex: Possible role of flocculus. J. Neurophysiol. 52: 1140-1153.

Maioli, C., and W. Precht (1984) The horizontal optokinetic nystagmus in the cat. Exp. Brain Res. 55: 494-506.

Marcotte, R. R., and B. V. Updyke (1982) Cortical visual areas of the cat project differentially onto the nuclei of the accessory optic component. Brain Res. 242: 205-217.

Melvill-Jones, G., and J. H. Milsum (1971) Frequency-response analysis of central vestibular unit activity resulting from rotational stimulation of the semicircular canals. J. Physiol. (Lond.) 219: 191-215.

Miles, F. A., and B. Eighmy (1980) Long-term adaptive changes in primatc vestibuloocular reflex. I. Behavioral observations. J. Neurophysiol. 5: 1406-1425.

Montarolo, P. G., W. Precht, and P. Strata (1981) Functional organization of the mechanisms subserving the optokinetic nystagmus in the cat. Neuroscience 6: 231-246.

Precht, W. (1982) Anatomical and functional organization of optokinetic pathways. In Functional Basis of Ocular Motility Disorders, G. Lennerstrand, D. S. Zee, and E. L. Keller, eds., pp. 291-302, Pergamon, Oxford, UK.

Raphan, T., and B. Cohen (1985) Velocity storage and the ocular response to multidimensional vestibular stimuli. In Adaptive Mechanisms in Gaze Control: Fact and Theories, A. Berthoz and G. Melvill Jones, eds., pp. 123-143, Elsevier/North-Holland, Amsterdam.

Robinson, D. A. (1977) Vestibular and optokinetic symbiosis: An examplc of explaining by modcling. In Control of Gaze by Brain Stem Neurons, Developments in Neuroscience, Vol. 1, R. Baker and A. Berthoz, eds., pp. 49-58, Elsevier/North-Holland, Amsterdam.

Robinson, D. A. (1981) Control of eye movements. In Handbook of Physiology, The Nervous Component, Vol. 2, Part 2, V. B. Brooks, ed., pp. 1275-1320, Williams \& Wilkins, Baltimore, MD.

Rosenquist, A. C. (1985) Connections of visual cortical areas in the cat. In Cerebral Cortex, A. Peters and E. G. Jones, eds., pp. 81-117, Plenum, New York.

Schoppmann, A. (1981) Projections from areas 17 and 18 of the visual cortex to the nucleus of the optic tract. Brain Res. 223: 1-17.

Segraves, M. A., and A. C. Rosenquist (1982) The afferent and efferent callosal connections of retinotopically defined areas in cat cortex. J. Neurosci. 2: 1090-1107.

Spear, P. D., and T. P. Baumann (1975) Receptive ficld charactcristics of single neurons in lateral suprasylvian visual area of the cat. $J$. Neurophysiol. 38: 1403-1420. 
Spear, P. D., and T. P. Baumann (1979) Effects of visual cortex rcmoval on receptive-field properties of neurons in lateral suprasylvian visual area of the cat. J. Neurophysiol. 42: 31-56.

Sprague, J. M., and T. H. Meikle, Jr. (1965) The role of the superior colliculus in visually guided behavior. Exp. Neurol. 11: 115-146.

Strong, N. P., R. Malach, P. Lee, and R. C. Sluyters (1984) Horizontal optokinetic nystagmus in the cat: Recovery from cortical lesions. Dev. Brain Res. 13: 179-192.

Tusa, R. J., L. A. Palmer, and A. C. Rosenquist (1981) Multiple cortical visual areas: Visual field topography in the cat. In Cortical
Sensory Organization, C. N. Woolscy, cd., pp. 1-31, Humana, Clifton, NJ.

Tusa, R. J., J. L. Demer, and S. J. Herdman (1983) Effects of visual cortical lesions on visual tracking in cats. Soc. Neurosci. Abstr. 9: 154.

Updyke, B. V. (1977) Topographic organization of the projections from cortical areas 17,18 , and 19 onto the thalamus, pretectum and superior colliculus in the cat. J. Comp. Neurol. 173: 81-122.

Ventre, J. (1985) Cortical control of oculomotor functions. I. Optokinetic nystagmus. Behav. Brain Res. 15: 211-226. 